\title{
Resolving the wave-particle-plasma interaction: advances in the diagnosis, interpretation and self-consistent modelling of waves, particles and the plasma configuration
}

\author{
M J Hole and M Fitzgerald \\ Research School of Physical Sciences and Engineering, Australian National University, Acton 0200, \\ ACT Australia \\ E-mail: matthew.hole@anu.edu.au
}

Received 22 October 2013, revised 18 February 2014

Accepted for publication 17 March 2014

Published 17 April 2014

\begin{abstract}
The purpose of this review is to present the state-of-the-art in diagnosis, interpretation and modelling of waves, particles and the magnetic configuration in fusion plasmas. Knowledge of the magnetic configuration underpins all confinement, stability and transport physics, as well as being an essential prerequisite for the inference of plasma parameters from many diagnostics. As the effect of fast particles become important enough to modify the macroscopic variables of the plasma, the macroscopic fluid equations for equilibrium need to be modified to encapsulate the effects of pressure anisotropy, particle and heat flow. We present a review of such modifications in tokamak geometry, and review probabilistic validation techniques of different equilibrium models. In the last decade new spectral tools have also emerged to characterize the linear behaviour of waves and wave-modes, such as SVD, Fourier-SVD, data-mining and the bispectrum. An emerging trend is the use of statistics to characterize the nonlinear wave population of the plasma from wave field data. Finally, progress is reported on developments in understanding the physics of wave-particle resonant interactions, and the emerging science of the wave-particle-plasma interaction.
\end{abstract}

Keywords: wave-particle interaction, resonance, equilibrium, diagnostics, validation classification

(Some figures may appear in colour only in the online journal)

\section{Introduction}

Instabilities such as Alfvén eigenmodes, driven by fast particles, are of programmatic concern as they can expel energetic ions from the plasma, thereby preventing heating by thermalization [1]. In addition, such energetic particles expelled can damage the first wall, and a fusion reactor can only tolerate fast particle losses of a few per cent [2]. Another motivation for the study of Alfvén eigenmodes is their potential use as a diagnostic for the plasma, particularly through the tool of magnetohydrodynamic (MHD) spectroscopy [3].

The over-arching purpose of this review is to present the state of the art in diagnosis, interpretation and modelling of waves, particles and the plasma configuration. The review is motivated by developments in diagnostic techniques, data analysis and signal processing tools, as well as new physics models that capture the impact of energetic particles on the plasma configuration. We commence with a treatment of 
advances in equilibrium modelling, which is the basis of all toroidal magnetic confinement. We discuss constraint of these models as well as developments in complex model validation. A large portion of the review is dedicated to wave characterization, in both the temporal and spatial domains. In addition to wavelet and Fourier transform techniques, we also discuss advances in mode number determination, the use of higher order spectral tools such as the bispectrum, and new approaches in analysing wave data using stochastic techniques, and thereby describing the nonlinear state of the plasma. Our work complements other recent summaries by focusing on equilibrium modelling and wave characterization. Breizman and Sharapov [1] focused on the development of plasma scenarios and novel energetic particle diagnostics, and nonlinear theory of energetic particle instabilities in the near threshold regime. For this reason, only a brief description of wave modes is supplied. Another focus of the review is to summarize developments in the physics of wave-particle resonant interactions, and to describe the emerging science of the wave-particle-plasma interaction, in which redistribution of energetic particles can lead to changes in the configuration, and thus the wave modes of the plasma. The manuscript is structured along these two themes: section 2 discusses developments in characterization, and section 3 discusses the wave-particle-plasma interaction. Finally, section 4 contains concluding remarks and challenges for the future.

\section{Characterization}

\subsection{Equilibrium}

Equilibrium reconstruction is a core topic in toroidal magnetic confinement fusion, as knowledge of the magnetic field structure underpins all confinement, stability and transport physics, as well as being essential prerequisite for the inference of plasma parameters from many diagnostics. As the effect of fast particles become important enough to modify the macroscopic variables of the plasma, the macroscopic fluid equations for equilibrium need to be modified to encapsulate the effects of pressure anisotropy, particle and heat flow. We present a review of such modifications in tokamak geometry. To make the review tractable, we have restricted attention to axis-symmetric plasmas.

2.1.1. Flow and anisotropy in ideal $M H D$. In time independent two-dimensional (2D) axisymmetric systems, the magnetic field and mass flow can each be expressed as

$$
\begin{gathered}
\boldsymbol{B}=\nabla \phi \times \nabla \psi+I(R, Z) \nabla \phi, \\
\rho \boldsymbol{v}=\nabla \phi \times \nabla \psi_{M}+I_{M}(R, Z) \nabla \phi,
\end{gathered}
$$

where we have used cylindrical coordinates $(R, \phi, Z)$ centred on the axis of the tokamak. Also, $I$ a poloidal current, $\psi$ is a poloidal magnetic field flux, related to the magnetic field components through

$$
B_{R}=-\frac{1}{R} \frac{\partial \psi}{\partial Z}, \quad B_{Z}=\frac{1}{R} \frac{\partial \psi}{\partial R},
$$

and the functions $\psi_{M}$ and $I_{M}$ are not yet defined. Substitution of equation (3) into the toroidal component of Ampere's law yields

$$
R^{2} \nabla \cdot\left(\frac{\nabla \psi}{R^{2}}\right)=-\mu_{0} R J_{\phi} .
$$

It is a well-known result that the existence of magnetic flux surfaces in guaranteed in 2D systems, and thus $\boldsymbol{B} \cdot \nabla \psi=0$ [4].

For the ideal MHD model, magnetic fields are frozen to the plasma and we may use equations (1) and (2) to identify two new flux functions constraining the poloidal and toroidal velocity

$$
\begin{gathered}
\psi_{M}^{\prime}(\psi)=\frac{\rho v_{\mathrm{p}}}{B_{\mathrm{p}}}, \\
\phi_{E}^{\prime}(\psi)=\frac{v_{\phi}}{R}-\frac{\psi_{M}^{\prime}(\psi) I}{\rho R^{2}}, \\
\boldsymbol{v}=\frac{\psi_{M}^{\prime}(\psi)}{\rho} \boldsymbol{B}-R \phi_{E}^{\prime}(\psi) \boldsymbol{e}_{\phi} .
\end{gathered}
$$

Most treatments of magnetic equilibrium are concerned with the second moment MHD equation

$$
\begin{aligned}
\rho(\boldsymbol{v} \cdot \nabla) \boldsymbol{v} & =-\nabla \cdot \overline{\boldsymbol{P}}+(\nabla \times \boldsymbol{B}) \times \boldsymbol{B}, \\
\overline{\boldsymbol{P}} & =p_{\perp} \overline{\boldsymbol{I}}+\Delta \boldsymbol{B} \boldsymbol{B}, \\
\Delta & \equiv\left(p_{\|}-p_{\perp}\right) / B^{2},
\end{aligned}
$$

which neglects viscosity by approximating the stress dyad $\overline{\boldsymbol{P}}$ as diagonalizable, or equivalently, that the stress is independent of gyro-angle. Although this expression for the stress dyad was originally obtained by Goldberger et al [5] one needs to make a further assumption about the insular independence of $p_{\|}$and $p_{\perp}$ before one can arrive at the 'CGL' or 'double-adiabatic model'. As far as equilibrium is concerned, the nature of the heat flow only becomes relevant when one considers non-zero plasma velocity or pressure anisotropy.

We must now obtain a set of scalar equations for the components of equation (8). The toroidal component of force balance yields a new flux function expression constraining the toroidal magnetic field

$$
\begin{gathered}
I_{M}(\psi)=\tau I-R^{2} \psi_{M}^{\prime} \phi_{E}^{\prime}, \\
\tau \equiv 1-\Delta-M_{\mathrm{p}}^{2}, \\
M_{\mathrm{p}}^{2} \equiv \frac{\left(\psi_{M}^{\prime}\right)^{2}}{\rho} .
\end{gathered}
$$

The remaining natural directions to obtain scalar equations are parallel to the field $\boldsymbol{B}$ and perpendicular with the field, in the direction of $\nabla \psi$. However, to proceed further, a thermodynamic statement must be made about how our pressure relates to the field and density by adding a thermodynamic closure. We consider the work done against the pressure to be equal to the change in energy per unit mass $U$ for a reversible process

$$
\bar{P}: \nabla v=\frac{p_{\|}}{\rho} \frac{\mathrm{d} \rho}{\mathrm{d} t}-\Delta B \frac{\mathrm{d} B}{\mathrm{~d} t}=\rho \frac{\mathrm{d} U}{\mathrm{~d} t},
$$


noting that energy is not lost through resistivity in ideal MHD. We may now proceed by specifying our convenient thermodynamic variables $U=U(\rho, B, \psi)$. The $\boldsymbol{B}$ component of force balance gives, after some rearranging,

$$
\begin{gathered}
H_{M}(\psi)=W(\rho, B, \psi)+\frac{1}{2}\left(\frac{\left(\psi_{M}^{\prime} B\right)^{2}}{\rho^{2}}-\left(R \phi_{E}^{\prime}\right)^{2}\right), \\
W(\rho, B, \psi) \equiv U+\frac{p_{\|}}{\rho},
\end{gathered}
$$

which is a Bernoulli type equation complete with a new flux function $H_{M}(\psi)$ analogous to the conserved energy along a streamline. Finally, we obtain a Grad-Shafranov (GS)-like equation from the $\nabla \psi$ component of force balance [6]

$$
\begin{aligned}
& \nabla \cdot\left[\tau\left(\frac{\nabla \psi}{R^{2}}\right)\right]=-\left.\frac{\partial p_{\|}}{\partial \psi}\right|_{B, \rho}-\rho H_{M}^{\prime}(\psi)+\left.\rho \frac{\partial W}{\partial \psi}\right|_{B, \rho} \\
& -I_{M}^{\prime}(\psi) \frac{I}{R^{2}}-\psi_{M}^{\prime \prime}(\psi) v \cdot B+R \rho v_{\phi} \phi_{E}^{\prime \prime}(\psi),
\end{aligned}
$$

subject to the integrability constraints

$$
\begin{gathered}
\left.\frac{\partial W}{\partial \rho}\right|_{B, \psi}=\left.\frac{1}{\rho} \frac{\partial p_{\|}}{\partial \rho}\right|_{B, \psi}, \\
\left.\frac{\partial W}{\partial B}\right|_{\rho, \psi}=\left.\frac{1}{\rho} \frac{\partial p_{\|}}{\partial B}\right|_{\rho, \psi}-\Delta \frac{B}{\rho} .
\end{gathered}
$$

Generally, equation (17) is an elliptic partial differential equation for physical equilibrium cases, and indeed, certain shock and instability phenomena are associated with transitions to a hyperbolic system, which occur because of poloidal rotation [7] or anisotropy [8] through the presence of $\tau$ in the differential operator. Here we will only discuss systems where the force-balance is elliptic.

The equilibrium is completely specified by the six free functions

$$
\left\{\left.\frac{\partial p_{\|}}{\partial \psi}\right|_{B, \rho}, I_{M}(\psi), H_{M}(\psi), \psi_{M}^{\prime}(\psi), \phi_{E}^{\prime}(\psi),\left.\frac{\partial W}{\partial \psi}\right|_{B, \rho}\right\},
$$

which respectively correspond to each physical assumption: parallel pressure, toroidal field, density on magnetic surfaces, poloidal flow, toroidal flow and thermodynamic closure. The perpendicular pressure is implicitly specified through the thermodynamic closure once the parallel pressure has been defined. Some choices for closure correspond to assumptions such as

$$
\begin{gathered}
\text { MHD }: p_{\|}=p_{\perp}=S(\psi) \rho^{\gamma}, \\
\text { CGL }: p_{\|}=S_{\|}(\psi) \frac{\rho^{3}}{B^{2}}, \\
p_{\perp}=S_{\perp}(\psi) \rho B,
\end{gathered}
$$

which can be inserted into equations (18) and (19) to obtain $W_{\mathrm{MHD}}(\rho, B, \psi)$ or $W_{\mathrm{CGL}}(\rho, B, \psi)$. Other expressions for $W$ are possible that can be derived from kinetic treatments such as the guiding centre plasma [9].
Simpler versions of the GS equation can be re-obtained as flow and anisotropy are removed from the model. For example, eliminating anisotropy using an adiabatic MHD model gives

$$
\begin{aligned}
\nabla \cdot & {\left[\left(1-M_{p}^{2}\right)\left(\frac{\nabla \psi}{R}\right)\right]=-\rho H_{M}^{\prime}(\psi)+\frac{\rho^{\gamma}}{\gamma-1} S^{\prime}(\psi) } \\
& -I_{M}^{\prime}(\psi) \frac{I}{R^{2}}-\psi_{M}^{\prime \prime}(\psi) \boldsymbol{v} \cdot \boldsymbol{B}+R \rho v_{\phi} \phi_{E}^{\prime \prime}(\psi),
\end{aligned}
$$

with the five free parameters

$$
\left\{I_{M}(\psi), H_{M}(\psi), \psi_{M}^{\prime}(\psi), \phi_{E}^{\prime}(\psi), S(\psi)\right\},
$$

and further eliminating toroidal and poloidal flow gives

$\nabla \cdot\left(\frac{\nabla \psi}{R^{2}}\right)=-\rho H_{M}^{\prime}(\psi)+\frac{\rho^{\gamma}}{\gamma-1} S^{\prime}(\psi)-I_{M}^{\prime}(\psi) \frac{I_{M}(\psi)}{R^{2}}$,

$$
H_{M}(\psi)=\frac{\gamma}{\gamma-1} \rho^{\gamma-1}(\psi) S(\psi),
$$

with three free functions. We see, by taking the full derivative with respect to $\psi$ in equation (27), that $H^{\prime}(\psi)$ can be inserted into equation (28) and made redundant

$$
\nabla \cdot\left(\frac{\nabla \psi}{R^{2}}\right)=-\left(\rho^{\gamma}(\psi) S(\psi)\right)^{\prime}-I_{M}^{\prime}(\psi) \frac{I_{M}(\psi)}{R^{2}},
$$

with the two free functions

$$
\left\{I \equiv I_{M}(\psi), p(\psi) \equiv \rho^{\gamma}(\psi) S(\psi)\right\} .
$$

Thus, we have arrived at the well-known GS equation for a static and isotropic plasma. The GS equation admits nested flux surface solutions, in which pressure surfaces coincide with surfaces of constant poloidal flux.

The most important difference between the modified and conventional GS equations relates to the magnetic properties of the pressure and poloidal current functions $p_{\|, \perp}(\rho, B, \psi)$ and $I(R, Z)$ through equations (15) and (11) respectively. If we examine the Bernoulli equation (equation (15)), we deduce that any dependence on $R$ or $B$ in the equation must be countered by an exactly opposing dependence through $\rho$ if $H_{M}(\psi)$ is to remain a strict flux function. Thus, it is clear that the mass density (and by implication, the pressure) cannot be a flux function in general. The same argument can be made for the toroidal force balance and the conditions under which $I_{M}(\psi)=I(\psi)$. The impact of beam direction relative to the field on perpendicular and parallel pressure profile shift was first characterized by Cooper et al [10]. For D-shaped cross-sections, Cooper found that the shift was a function of the broadness of the pressure profile. The most significant result was found for broad-pressure-profile equilibria and perpendicular beam injection, where it was found that $p_{\|}$ contours undergo a significant shift away from the outer edge of the device with respect to the flux surfaces.

Constraints to experiment can be done for the modified GS equation in a very similar fashion as for the static version. Equations (11) and (4) can be constrained by external magnetics such as flux loops magnetic pickup coils. Accurate representation of the internal magnetic topology requires internal constraints such as motional Stark effect (MSE) and 
Faraday rotation. Additionally, the internal magnetic topology may be further constrained by locating the position of various shear surfaces implied by the observed MHD activity. It is also possible to infer properties of $\nabla p_{\perp}$ from the diamagnetic loop signal [11].

The moments of the particle distribution functions provide the remaining density, rotation and pressure constraints required. These may be acquired spectroscopically but are typically incomplete in energy, location and species without further assumptions. Indeed, many of the forward models which are used to measure the distribution function make prior assumptions on their approximate form. Ultimately, an integrated modelling approach is required utilizing transport calculations such as TRANSP [12] where the various input and output torques and powers from NBI and/or resonant heating are balanced.

An implementation of a bi-Maxwellian kinetic closure neglecting poloidal rotation has been recently developed by Fitzgerald et al [13]. The code, EFIT TENSOR, is a modification of the existing force balance solver EFIT++, which is constrained to external magnetics vacuum toroidal field, flux loops, magnetic probes, plasma current, poloidal field coils, safety factor on axis $q_{0}$, static and rotational pressure approximations, $B$ components, diamagnetic flux, boundary, equal $\psi$ surfaces, and MSE. To this set of constraints, EFIT TENSOR adds kinetic constraints of $p_{\|}, p_{\perp}$ and a toroidal flow profile. Both EFIT and EFIT TENSOR codes are equilibrium reconstruction codes, which find least squares fit solutions to the data for GS, and flow and anisotropy modified GS equations. To demonstrate the impact of full order flow and anisotropy, Fitzgerald et al have compared reconstructions using ideal MHD, and ideal MHD equilibrium with flow and anisotropy for MAST discharge \#18696 at $290 \mathrm{~ms}$. Figure 1 shows the difference in radial force balance. TRANSP, which uses the rotational pressure assumption, underestimates the plasma pressure contribution either side of the magnetic axis, and the MHD equilibrium with flow and anisotropy is not satisfied away from the magnetic axis. At maximum, the discrepancy is of order $35 \%$, revealing that the effect is significant.

\subsubsection{Probabilistic methods. Bayesian inference offers an} alternate approach to equilibrium modelling in fusion plasmas [14-18], and a pathway to validate different equilibrium model descriptions [19,20]. In Bayesian inference, an initial prior probability distribution function (pdf), $P(\boldsymbol{I})$, for the plasma parameters $I$ is specified, which is then updated by taking into account information that the measurements $D$ provide through the likelihood pdf $P(\boldsymbol{D} \mid \boldsymbol{I})$. The result is the posterior distribution $P(\boldsymbol{I} \mid \boldsymbol{D})$ given by Bayes formula

$$
P(\boldsymbol{I} \mid \boldsymbol{D})=P(\boldsymbol{D} \mid \boldsymbol{I}) P(\boldsymbol{I}) / P(\boldsymbol{D}) .
$$

where $P(D)$ is a renormalization constant of the posterior called the evidence. The advantage of the Bayesian approach over traditional inversion techniques is two-fold: (i) prior knowledge, including known parameter inter-dependencies is made explicit, and (ii) as the formulation is probabilistic, random errors, systematic uncertainties and instrumental bias
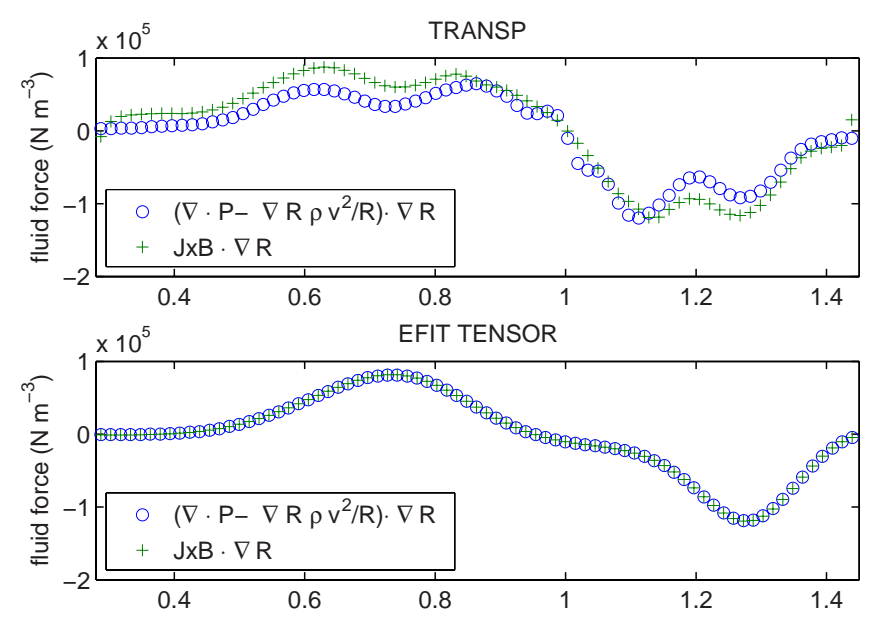

Figure 1. Finite difference radial force balance for profiles produced by TRANSP and EFIT TENSOR, with the inclusion of full order flow and anisotropy for MAST\#18696 at $290 \mathrm{~ms}$. Reproduced with permission from figure 6 of Fitzgerald et al [13]. Copyright IAEA 2013.

are integral part of the analysis rather than an afterthought. Hole et al have implemented Bayesian inversion on MAST using the MINERVA framework [21]. Within this framework, probabilistic graphical models are used to project the dependence of the posterior distribution function on the prior, the data, and the likelihood. An advantage of this approach is that it visualizes the complex interdependency between data and model, and thus expedites model development. The techniques of Bayesian inference have also been inverted to provide a tool to check data consistency [22].

Hole et al have developed Bayesian inference techniques for fusion plasmas that combine information from a wealth of diagnostics to enable probabilistic calculation of plasma configuration, [18] provide automatic identification of faulty diagnostics, [22] and developed a validation tool for generalized force balance models [19]. Critically, Bayesian techniques propagate experimental uncertainty correctly, and enable the relative uncertainty between acceptable physical models to be quantified. In von Nessi et al [20], a new method, based on Bayesian analysis, is presented which unifies the inference of plasma equilibria parameters in a tokamak with the ability to quantify differences between inferred equilibria and GS force balance solutions. At the heart of this technique is the new concept of weak observation, which allows multiple forward models to be associated with a single diagnostic observation. This new idea subsequently provides a means by which the space of GS solutions can be efficiently characterized via a prior distribution. The posterior evidence (a normalization constant of the inferred posterior distribution) is also inferred in the analysis and is used as a proxy for determining how relatively close inferred equilibria are to force balance for different discharges/times.

Figure 2 shows expectation values of the toroidal current density inferred from $(a)$ a toroidal current beam model, $(b)$ a GS constraint, in which $J_{\phi}$ is computed from GS from a $\psi$ surface, together with fits to the pressure and toroidal flux function, and forward models for magnetics, total plasma current and MSE predictions, and $(c)$ the difference between 


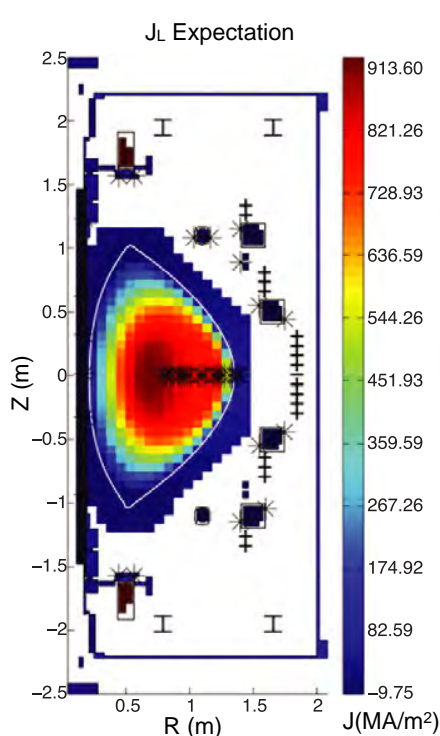

(a)

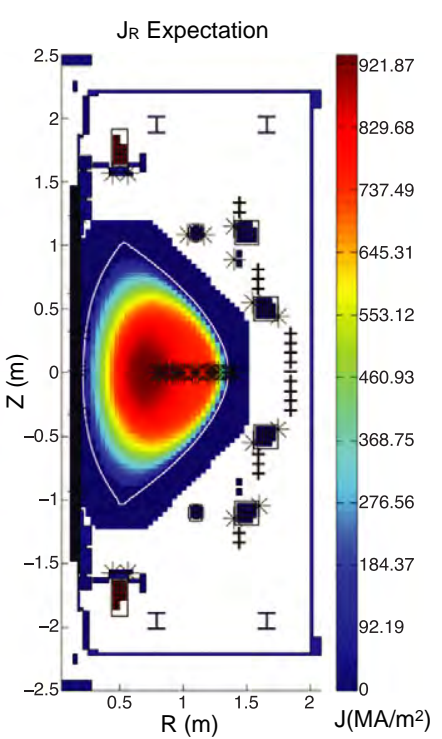

(b)

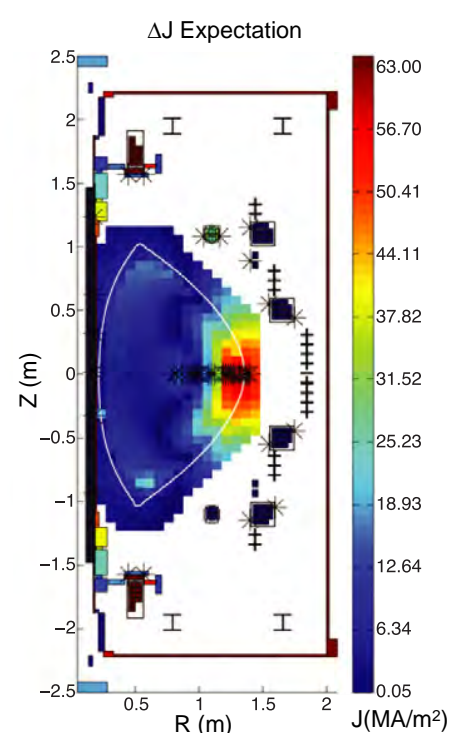

(c)

Figure 2. Expectation values of $J(R, Z), J_{G S}(R, Z)$ and $\Delta J(R, Z)$ inferred for MAST discharge \#22254 at $350 \mathrm{~ms}$, as calculated from 1800 samples of the posterior, using pickup coils, flux loops, MSE and Rogowski coil data. The inferred last closed flux surface is indicated in white on each figure. Flux loop locations are indicated by stars outside the plasma region; the position and orientation of pickup coils are indicated via heavy bars on the out-board edge of the first wall and as a vertically oriented column line along the solenoid; and MSE observation positions are indicated by the stars across the mid-plane inside the plasma region. Panel $(a)$ shows $J(R, Z)$ current density data, with the current densities in $(b)$ reflecting that of $J_{G S}(R, Z)$. Note that the number and size of beams representing $J(R, Z)$ and $J_{G S}(R, Z)$ are allowed to differ in BEAST inferences. $(c)$ shows the magnitude of the current density difference as averaged across each $2 \mathrm{D}$ rectangular step corresponding to $J(R, Z)$. Reproduced with permission from figure 2 of [20].

the two. The difference in $J_{\phi}$ can give some indication to physical effects neglected in the GS equation, and/or reflect diagnostic disagreement. In this case the discrepancy is largest at the outboard mid-plane, and of order of $10 \%$. Using nested sampling, it is possible to integrate over the evidence, and thus compute $\ln (P(D))$ of the inferred hyperparameter, $\sigma^{2}$, which is the average current variance between GS and toroidal current beam values. The smaller the value of $\ln (P(D))$, the larger the degree of freedom necessary to predict diagnostic observations relative to other cases. For 22254 at $350 \mathrm{~ms} \sigma_{*}^{2}=9.461 \times 10^{-3} \pm 1.2 \times 10^{-5}(\mathrm{kA})^{2}$ and $\ln (P(D))=765$, while $\sigma_{*}^{2}=0.203381 \pm 1.59 \times 10-4(\mathrm{kA})^{2}$ and $\ln (P(D))=-39$ for adjacent discharge \#24600 at $265 \mathrm{~ms}$. This meant \#22254 was much closer to GS, and/or had fewer diagnostics in conflict, than \#24600.

\subsection{Wave theory}

Most understanding of electromagnetic waves in fusion plasmas is based on a wave perturbation analysis of the ideal MHD model, which describes a plasma in local thermal equilibrium. As the system is periodic in both toroidal and poloidal directions, the perturbation is of the form $\exp (i(m \theta+$ $n \phi-\omega t)$ ), with $m, n$ the poloidal and toroidal mode number, respectively, and $\theta$ and $\phi$ the poloidal and toroidal angle, respectively, $\omega$ the frequency and $t$, time. Thermal plasmas can support a wide range of electromagnetic waves, many of which can be driven unstable by energetic particles. Experimentally, the most prolific wave is the shear Alfvén wave, which represents a balance between plasma inertia and field line tension. In cylindrical plasmas, shear Alfvén waves of finite radial extent cannot persist without coupling to adjacent radial wave packets. The phenomenon, known as phase mixing, leads to damping of continuum modes. The inclusion of symmetrybreaking geometric effects can, however, break degeneracy's, leading to band gaps in the continuum, where damping due to phase mixing is much reduced, and thus modes in the gap can be driven unstable. The first such bandgap, corresponding to 'elliptical Alfvén eigenmodes' (EAEs) was discovered by Dewar [23]. In tokamak plasmas, the most prolific of these band gap modes are toroidal Alfvén eigenmodes (TAEs) [24].

In addition to perturbative modes, which are modes of the background thermal (i.e. Maxwellian) plasma driven unstable by the energetic populations, there also exist non-perturbative modes (energetic particle modes or EPMs) which exist only in the presence of an energetic population. EPMs have frequencies characteristic of energetic-particle motion, such as transit, bounce, and precession frequencies. Figure 3 is a schematic of the energetic-particle driven zoo of normal modes, illustrating both the range of modes that can be driven, and their spatial localization [25].

2.2.1. Wave measurements. One of the simplest and most effective diagnostics of wave activity are external Mirnov coils, which are solenoidal coils that measure magnetic field oscillations. Although the basic principles are well understood, optimizing the coil design requires careful analysis. Recent treatments of the design of high frequency coils include: Bak et al [26], who developed a Mirnov coil system for KSTAR with self-resonant frequency of $12 \mathrm{MHz}$, which was downshifted to $1 \mathrm{MHz}$ due to unmatched signal cabling; Hole et al [27], which designed and built a high 


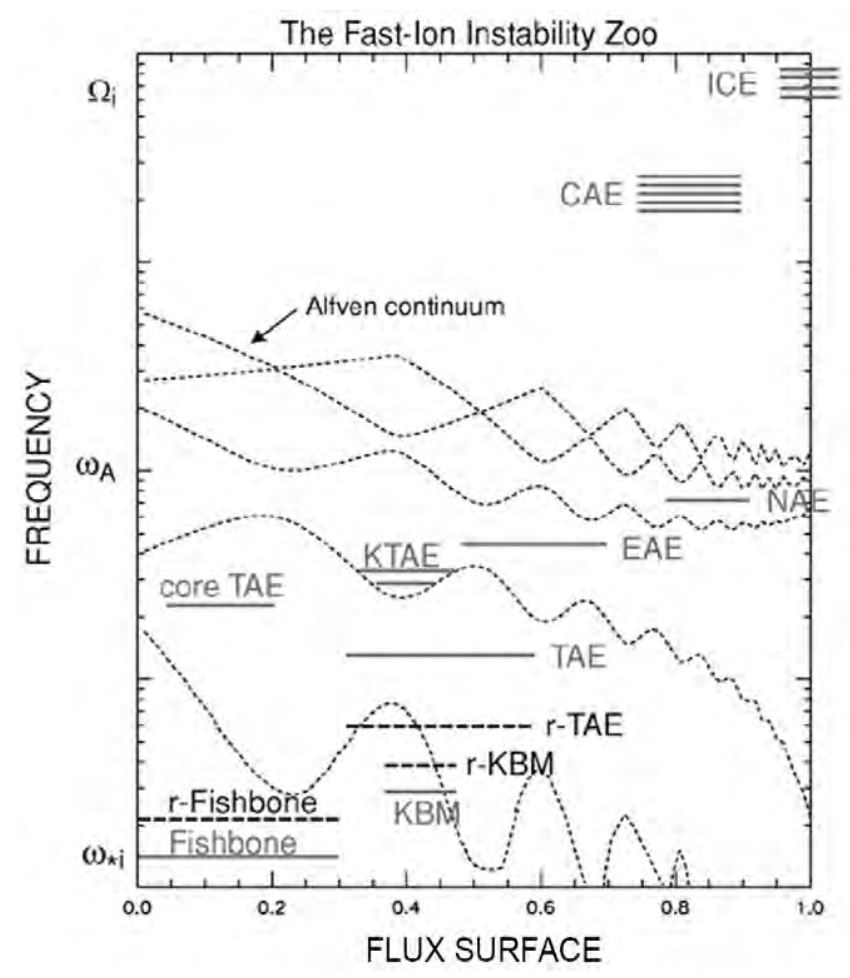

Figure 3. Normal modes of a tokamak: $\Omega_{i}, \omega_{A}$, and $\omega_{* i}$ are the ion cyclotron, Alfvén and ion diamagnetic drift frequencies. Modes and their radial localization are shown as horizontal lines. Reproduced with permission from Heidbrink [25]. Copyright 2002 AIP Publishing LLC.

frequency Mirnov array for MAST with a self-resonant frequency above $5 \mathrm{MHz}$ and maximized voltage output at $100 \mathrm{kHz}$; and Testa and Albergante [28], who present a prototype study of a high frequency magnetic coils for ITER. External magnetic coils are an important tool for the detection of magnetic modes which have a significant perturbation near the plasma edge which couples to external vacuum. Such modes typically have low toroidal mode numbers.

The in situ shielding requirements of magnetic coils can also place limitations on their frequency response, ranging from mild to potentially severe. The OMAHA coils in MAST for example are mounted at the end of a $30 \mathrm{~cm}$ long, $17.5 \mathrm{~mm}$ radius cylindrical $\mathrm{Al}_{2} \mathrm{O}_{3}$ ceramic test tube with $0.2 \mathrm{~mm}$ layer of colloidal graphite, which was bolted to the vessel wall. This largely eliminated the effect of currents induced in the wall. In ITER, the coils will be placed behind the blanket modules [29]. Eddy currents have decay times in the range $7-17 \mathrm{~ms}$ [30]. If the blanket module were contiguous, signals would be limited to well below $200 \mathrm{~Hz}$. Fortunately, Mirnov coils will be positioned under poloidal gaps between adjacent blanket modules [29]. For the high mode frequencies expected in the interval $10-500 \mathrm{kHz}$ the gap can be considered as a wave guide operating in the cutoff mode. For the gap dimensions of the ITER blanket modules the cutoff frequency for the TE10 mode is $38 \mathrm{MHz}$, and the attenuation below $500 \mathrm{kHz}$ is frequency independent, and approximately 0.48 . The effect of blanket shielding on phase is currently being examined [31].

Microwave interferometry/reflectometry provides measurements of perturbed electron density, and thereby offers a tool to characterize modes internal to the plasma [32]. A microwave beam propagating through the plasma undergoes a change in amplitude and a shift in phase due to the variation of the refractive index caused by the electron density. Frequencies above the mode cutoff frequency will propagate across the plasma and be reflected from the inner wall of the plasma. In the cold plasma approximation the 'O' mode cutoff is $\omega_{\mathrm{pe}}=\left(n_{e} e^{2} / \epsilon_{0} m e\right)^{1 / 2}$, so for given microwave frequency the diagnostic will act as an interferometer up to a cutoff density. At higher frequencies the microwaves will be reflected within the plasma, and the diagnostic operates as a reflectometer. The signals associated with changes in the density, $\delta n$, are obtained from the amplitude and the change of the phase of microwave beams by comparing the beam propagating through the plasma with the reference beam outside the plasma. These techniques provide line integrated measurements of density perturbations in frequency ranges comparable to Alfvén wave mode activity. Sharapov et al [33] have monitored Alfvén cascade activity on JET using an interferometer. The value of the diagnostic is in providing clear indication of the density of states of rational surfaces and reliable identification of integer $q_{\min }(t)$ events. Figure 4 shows an example of interferometry measurements in JET, together with Mirnov coil measurements. In contrast to the interferometer measurements, which are line integrated across the chord of the beam through the plasma, Mirnov coils measure external oscillations at the probe location, and so are not sensitive to internal plasma oscillations. Unlike interferometry however, a toroidal/poloidal array of Mirnov coils does provide toroidal/poloidal mode numbers. Similar results to Sharapov et al have been obtained by Zeeland et al in DIII-D plasmas [34]. Reflectometry measurements of mode structures have been reported in JET [35] and DIII-D plasmas [36]. Orsitto et al [37] provides a wider review of requirements for fast particle measurements on ITER and candidate measurement techniques.

The canonical approach to identify wave activity from time series data is through the Fourier transform [38]. This integral transform

$$
x(t)=\frac{1}{\sqrt{2 \pi}} \int_{-\infty}^{\infty} F(\omega) \mathrm{e}^{\mathrm{i} \omega t} \mathrm{~d} \omega,
$$

expresses the signal $x(t)$ in terms of a complex function $F(\omega)$ whose magnitude represents the correlation of $x(t)$ with a wave $\mathrm{e}^{\mathrm{i} \omega x+i \chi}$ with phase $\chi$ chosen to maximize $F(\omega)$, and the phase of $F(\omega)$ is $\chi$. The variable $\omega$ is angular frequency. The short time base Fourier transform is often used, with $N_{s}$ samples, to resolve the temporal variation of spectral components. In most laboratory plasma experiments, temporal data is regularly sampled with sampling time spacing $\delta t_{s}$, leading to a linear Fourier array of frequencies ranging from $f_{s} /(2 N) \leqslant f \leqslant$ $f_{s} / 2$, with the sampling frequency $f_{s}=1 / \delta t_{s}$. A spectrogram is a short time Fourier transform, in which a windowing function may be applied to remove or limit artefacts [38].

Wavelet analysis is a generalization of short time Fourier transform, and is commonly used in turbulence analyses [39]. A wavelet is any function $\Psi(t)$, whose Fourier transform $\Psi(\omega)$ satisfies the wavelet admissibility condition:

$$
c_{\Psi}=\int_{-\infty}^{\infty}|\Psi(\omega)|^{2}|\omega|^{-1} \mathrm{~d} \omega<\infty,
$$




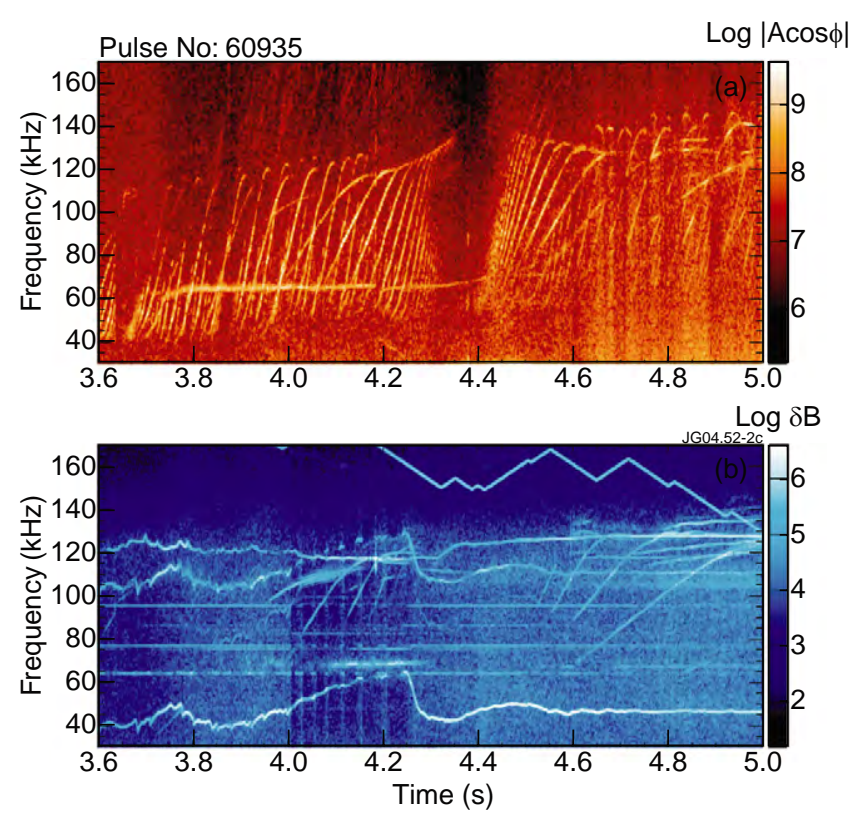

Figure 4. Fourier spectrograms showing Alfvén cascades with different toroidal and poloidal mode numbers JET discharge \#60935. The top panel shows interferometry measurements with a microwave beam of $45.2 \mathrm{GHz}$. The bottom panel shows measurements with external magnetic pick-up coil. Reproduced with permission from figure 2 of Sharapov et al [33]. Copyright 2003 American Physical Society.

such that the corresponding wavelet family is obtained by means of the scale length parameter $a$,

$$
\Psi_{a, b}(t)=\left(1 / a^{p}\right) \Psi((t-b) / a),
$$

where $b$ is the translation and $a$ is the scale. The wavelet transform of $x(t)$ is then given by

$$
W_{x}(a, b)=\int f(t) \Psi_{a, b}(t) \mathrm{d} t,
$$

and the commonly used scalagram $S(a, b)=\left|W_{x}(a, b)\right|^{2}$, where time and frequency are given by $t=b$ and $f=F_{c} / a$. A common choice for the wavelet in plasma physics is the complex Moret wavelet, which is a sinusoid within a Gaussian envelope:

$$
\Psi(\tau)=\frac{1}{\sqrt{\pi F_{\mathrm{b}}}} \exp \left(i 2 \pi F_{c} \tau\right) \exp \left(-\frac{\tau^{2}}{F_{\mathrm{b}}}\right),
$$

where $F_{c}$ is the centre frequency and $\sqrt{F_{\mathrm{b}}}$ a decay time scale of the wave packet [39]. The wavelet transform can be considered a short time Fourier transform with a windowing function, whose window length decreases inversely with frequency such that the number of wavelets is fixed.

A third approach to characterizing the spectral content of non-stationary signals is use of the quadratic time-frequency distributions introduced by Cohen in the context of quantum mechanics [40]. Each member of this distribution class is given by

$C_{x}(t, \omega ; \phi)=\frac{1}{2 \pi}$

$\times \iiint \mathrm{e}^{j(\zeta \mu-\tau \omega-\zeta t)} \phi(\zeta, t) x(\mu+\tau / 2) x^{*}(\mu-\tau / 2) \mathrm{d} \mu \mathrm{d} \tau \mathrm{d} \zeta$, where $x(\mu)$ is the time signal, $x^{*}(\mu)$ its complex conjugate, and $\phi$ a kernel function. The Choi-Williams distribution [41] is a particular choice of exponential kernel function, for which

$$
\phi(\zeta, \tau)=\exp \left(-\zeta^{2} \tau^{2} / \sigma\right)
$$

with $\sigma>0$ is a scalar factor that represents the localization of the wave packet. For large values of $\sigma, C_{x}(t, \omega)$ has very good frequency resolution, but poor artefact resolution. Reduction of artefacts is achieved by reducing $\sigma$, with some loss of time-frequency resolution [42]. Figure 5 shows the spectrogram, the scalogram or wavelet and analysis and ChoiWilliams distribution of JET \#55976 [42]. The figure shows type I edge localized mode (ELM) precursors at $15 \mathrm{kHz}$, interrupting wash-board modes at $25-50 \mathrm{kHz}$. The scalagram has better frequency resolution than the spectrogram, but poorer temporal resolution. In contrast, the Choi-Williams distribution achieves better temporal and frequency resolution than either. While the difference in analysed signal is particularly clear in this example, the use of spectrograms is much more widespread, both because of simplicity, and because it is possible to achieve a comparable result to the Choi-Williams distribution across a narrow band-width.

2.2.2. Mode numbers. In a configuration with periodicity such as toroidal magnetic confinement wave numbers become discretized, and so the problem of inference of spatial wave numbers is replaced with inference of mode numbers. The simplest approach to measuring phase variation is through the use of cross-correlation techniques. These measure the phase difference between two signals $f_{1}(t)$ and $f_{2}(t)$ through

$$
r_{x y}(\tau)=\lim _{T_{0} \rightarrow \infty} \frac{1}{T_{0}} \int_{-T_{0} / 2}^{T_{0} / 2} f_{1}(t) f_{2}(t+\tau) \mathrm{d} t .
$$

The frequency analogue of this approach is cross-spectral techniques, in which the complex cross-spectrum a probe pair is computed using

$$
C_{12}(\omega)=\left\langle F_{1}(\omega) F_{2}^{*}(\omega)\right\rangle,
$$

where $F_{1}$ and $F_{2}$ are the complex Fourier transforms of the digitized coil magnetic signal of coils 1 and 2 , and \langle\rangle denotes averaging in the frequency domain over an interval $\delta \omega$ centred about $\omega$. The phase spectrum

$$
\Theta_{12}=\tan ^{-1}\left\{\operatorname{Im}\left[C_{12}(\omega)\right] / \operatorname{Re}\left[C_{12}(\omega)\right]\right\},
$$

gives the phase difference between the probes as a function of frequency. The toroidal mode number is then be estimated by

$$
n(\omega)=\Theta_{12}(\omega) / \delta \phi,
$$

rounded to the nearest integer, with $\delta \phi=\phi_{1}-\phi_{2}$, the spacing between the coils.

An early approach to determining mode numbers from an array of data is through the use of a phase time diagram. In this approach points of common phase on a phase time diagram and a straight-line best fit performed. Poloidal mode numbers may be similarly computed with the poloidal angle computed by 

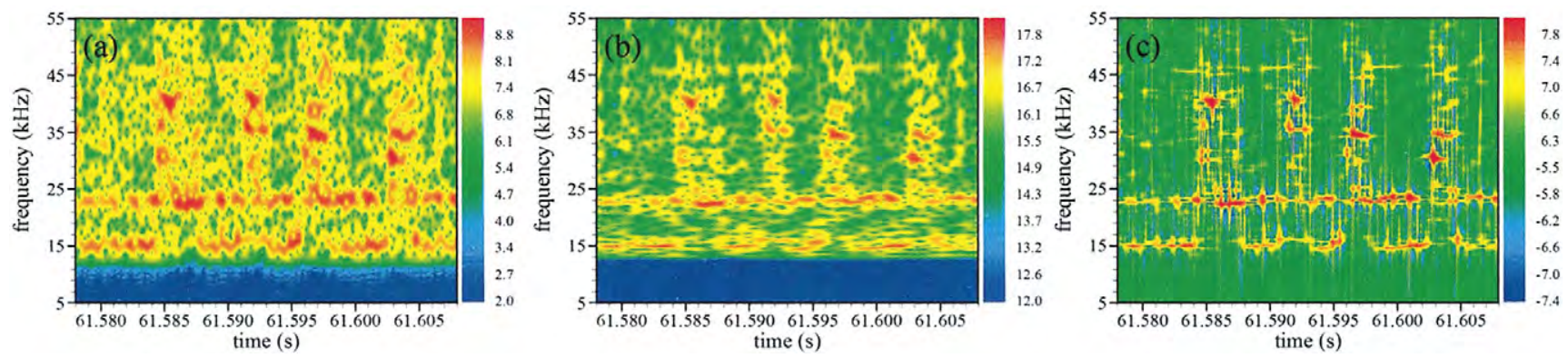

Figure 5. Analysis of a magnetic pickup coil signal (JET pulse 55976), using $(a)$ the spectrogram with $l=255,(b)$ the scalogram with $F_{\mathrm{c}}=1 \mathrm{~Hz}$ and $F_{\mathrm{b}}=200 \mathrm{~s}^{2}$, and $(c)$ the Choi-Williams distribution with $l_{\tau}=2047, l_{\mu}=255$ and $\sigma=0.5$. Reproduced with permission from figure 1 of Figueiredo and Nave [42]. Copyright 2004 AIP Publishing LLC.

the Merezkhin expression [43, 44], which accounts for toroidal curvature to lowest non-vanishing order in inverse aspect ratio $\epsilon$. Over time, the Merezhkin expression has increased in order, capturing the effects of toroidal geometry and plasma shaping to at least $O\left(\epsilon^{3}\right)$ [45]. Indeed, if the equilibrium magnetic field solution is available, the straight-field-line poloidal angle is available to arbitrary accuracy [46].

More sophisticated approaches, which harness and exploit the sparse and often irregularly spaced diagnostics include singular value decomposition (SVD)[47-50], FourierSVD [51], and the code and methodology SparSpec [52]. Nardone [47] (and subsequently others [48-50]) have applied principal component analysis to the time traces of spatially resolved magnetic and soft $x$-ray data channels, enabling the simultaneous use of information from the entire detector array. The method involves construction of a $N_{\mathrm{s}} \times N$ rectangular matrix

$$
\boldsymbol{X}=\frac{1}{\sqrt{N}}\left(\begin{array}{ccc}
x(0) & \cdots & x_{N}(0) \\
x\left(\Delta t_{\mathrm{s}}\right) & \cdots & x_{N}\left(\Delta t_{\mathrm{s}}\right) \\
\vdots & \vdots & \vdots \\
x\left(\left(N_{\mathrm{s}}-1\right) \Delta t_{\mathrm{s}}\right) & \cdots & x_{N}\left(N_{\mathrm{s}}-1\right)\left(\Delta t_{\mathrm{s}}\right)
\end{array}\right),
$$

where $N_{\mathrm{s}}$ is the number of samples, $\Delta t_{\mathrm{s}}$ the sampling time, and $N$ the number of channels. SVD matrix inversion [53] expresses $\boldsymbol{X}$ in terms of the product

$$
\boldsymbol{X}=\boldsymbol{U}_{x} \cdot \operatorname{diag}\left(w_{x, i}\right) \cdot \boldsymbol{V}_{x}^{T},
$$

where $\boldsymbol{U}_{x}$ and $\boldsymbol{V}_{x}$ are column-orthonormal $N_{\mathrm{s}} \times N$ and $N \times N$ matrices, respectively, and $w_{x, i}$ are the singular values of $\boldsymbol{X}$. The column vectors $\boldsymbol{v}_{x}^{(j)}=V_{x, i j}$ are the principal axes, and form the orthonormal basis on which the signal is spatially decomposed. The projections of $\boldsymbol{X}$ along $V_{x}$ (i.e. the product $\boldsymbol{U}_{x} \cdot \operatorname{diag}\left(w_{x, i}\right)$ are the principal components, and give the time evolution of the signal along the corresponding principal axis. To extract the mode numbers, the principal axes must either be fitted to a Fourier spatial basis, or a counting operation applied to the zero crossings of their spatial projection.

In fusion, the terms principle component analysis, SVD and bi-orthogonal decomposition have been used interchangeably [54]. Principal component analysis is very closely related to other techniques in different fields, including: the discrete Karhunen-Loéve transform and the
SVD method in signal processing [55], and proper orthogonal decomposition in computational science [56].

Fourier-SVD [51] is a procedure whereby the rows of $\boldsymbol{X}$ are replaced by their Fourier transform, and a basis imposed for the Fourier weights. For a coil located at angle $\phi_{k}$, the integrands $F_{k}(\omega)$ in equation (31) can be written

$$
F_{k}=\sum_{i=1}^{M} \alpha_{\mathrm{i}} \mathrm{e}^{j n_{i} \phi_{k}}
$$

At a particular frequency each measurement provides two constraints via the complex Fourier transform $F_{k}$. The measurement is matched to a set of modes each one of which has three unknowns: magnitude, phase and eigenmode number. Equation (44) can be rewritten as

$$
\boldsymbol{F}=\gamma \cdot \boldsymbol{\alpha}
$$

for $N$ coils, with

$$
\begin{gathered}
\boldsymbol{F}=\left(\begin{array}{l}
F_{1} \\
F_{2} \\
\vdots \\
F_{N}
\end{array}\right), \gamma=\left(\begin{array}{lll}
\mathrm{e}^{\mathrm{j} n_{1} \phi_{1}} & \cdots & \mathrm{e}^{\mathrm{j} n_{M} \phi_{1}} \\
\mathrm{e}^{\mathrm{j} n_{1} \phi 2} & \cdots & \mathrm{e}^{\mathrm{j} n_{M} \phi_{2}} \\
\vdots & & \vdots \\
\mathrm{e}^{\mathrm{j} n_{1} \phi_{N}} & \cdots & \mathrm{e}^{\mathrm{j} n_{M} \phi_{N}}
\end{array}\right), \\
\boldsymbol{\alpha}=\left(\begin{array}{l}
\alpha_{1} \\
\vdots \\
\alpha_{M}
\end{array}\right),
\end{gathered}
$$

where $M$ is the number of distinct eigenmodes in the plasma at angular frequency $\omega$. Equation (45) comprises $2 N$ equations in the $3 M$ unknowns: the eigenmode numbers $n_{1}, \ldots, n_{M}$; the mode amplitudes $\left|\alpha_{1}\right|, \ldots,\left|\alpha_{M}\right|$; and mode phases $\tan ^{-1}\left(\alpha_{1}^{i} / \alpha_{1}^{r}\right), \ldots, \tan ^{-1}\left(\alpha_{M}^{i} / \alpha_{M}^{r}\right)$. Solutions of equation (45) thus yield simultaneous extraction and hence identification of up to $2 N / 3$ distinct eigenmodes. Fourier-SVD finds the best fitting mode combination by obtaining the inverse of $\gamma$ through SVD inversion, subject to the overall minimization of the residual

$$
r=|\gamma \cdot \boldsymbol{\alpha}-\boldsymbol{F}| /|\boldsymbol{F}|
$$

Fourier-SVD has been inverted to determine an optimal configuration for magnetic coils [27].

A similar technique for the inference of frequency spectrum comes from astronomy [52], where the objective is inferring spectral content using a minimum information 
approach. Like Fourier-SVD, the technique is well suited when sampling is sparse and irregular. In this approach data $y_{n}$ is modelled by

$$
y_{n}=\sum_{k=-K}^{K} x_{k} \exp \left(j 2 \pi \frac{k}{K} f_{\max } t_{n}\right)+\epsilon_{n},
$$

with $x_{k}$ are unknown spectral amplitudes and $\epsilon_{n}$ is a perturbation term that stands for model errors and observation noise. In matrix-vector form equation (47) can be rewritten

$$
\boldsymbol{y}=\boldsymbol{W} \cdot \boldsymbol{x}+\epsilon,
$$

where $\boldsymbol{y}=\left[y_{1} \ldots y_{N}\right]^{T}, \boldsymbol{x}=\left[x_{-K} \ldots x_{K}\right]^{T}, \boldsymbol{\epsilon}=\left[\epsilon_{1} \ldots \epsilon_{N}\right]^{T}$, and the matrix $\boldsymbol{W}=\left\{\exp \left(j 2 \pi f_{k} t_{n}\right)\right\}_{k=-K \ldots K, n=1 \ldots N}$. The method has been adapted to infer mode numbers by Klein et al [57], who replace $\boldsymbol{y}$ with the Fourier transform $\boldsymbol{F}$ in equation (44), and $\boldsymbol{W}$ with $\gamma$ in equation (45). The difference between the two approaches is the minimization criterion. In SparSpec it is

$$
J(x)=\frac{1}{2}\|\boldsymbol{y}-\boldsymbol{W} \boldsymbol{x}\|+\frac{\lambda}{\lambda_{\text {Max }}} \sum_{k=-K}^{K}\left|x_{k}\right|,
$$

with $\lambda$ a hyper-parameter whose magnitude controls the number of modes that are fitted to the data.

Figure 6 shows Fourier-SVD and SparSpec fits to mode activity in MAST and JET magnetic coil data, respectively. In panel (a) a Fourier-SVD fit of low $n$ modes, separated by toroidal rotation, together with high frequency modes of opposite $n$ sign, believed to be aliased compressional Alfvén eigenmodes (CAEs) [58]. A filter has been applied to suppress mode fits if the signal/noise ratio is below five. In panel $(b)$, a SparSpec fit to modes reveals a grand cascade of reverse shear Alfvén eigenmodes, with sequentially lower toroidal mode number.

The difference between Fourier-SVD and SparSpec lies in the choice of number of modes, and normalization. In FourierSVD the spatial mode basis is fit at every frequency, and may account for multiple modes at the same frequency, i.e. $M>1$. SparSpec however does not fit the spatial mode basis at every frequency: the number of modes is implicitly set through the hyperparameter $\lambda$. SparSpec is routinely used with less than ten modes. Fourier-SVD uses the $L^{1}$ norm of $|\gamma \cdot \boldsymbol{\alpha}-\boldsymbol{F}| /|\boldsymbol{F}|$, while SparSpec uses a norm that is $L^{2}$ in $\boldsymbol{y}-\boldsymbol{W} \cdot \boldsymbol{x}$ and $L^{1}$ in $\boldsymbol{x}$. The latter choice of normalization enables fine control over the number of modes fitted: for a given value of $\lambda$ the mode basis is minimized. As implemented by Klein et al the choice of $\lambda$ is empirical, and it is selected as small as possible while 'still avoiding erratic or unlikely results in the output'. Fourier-SVD has established the number of meaningful modes fit by computing the fit of noise, and comparing the residue of the signal fit to that of noise. Plasma signals are identified if the probability that noise could generate the same residue and mode number, or same amplitude falls below $1 \%$ confidence. Practically, the two techniques are both routinely implemented for fast analysis. As SparSpec solves for a smaller basis set, it thus may be more amenable to real time mode identification. Fourier-SVD is capable of resolving multiple modes at the same frequency.
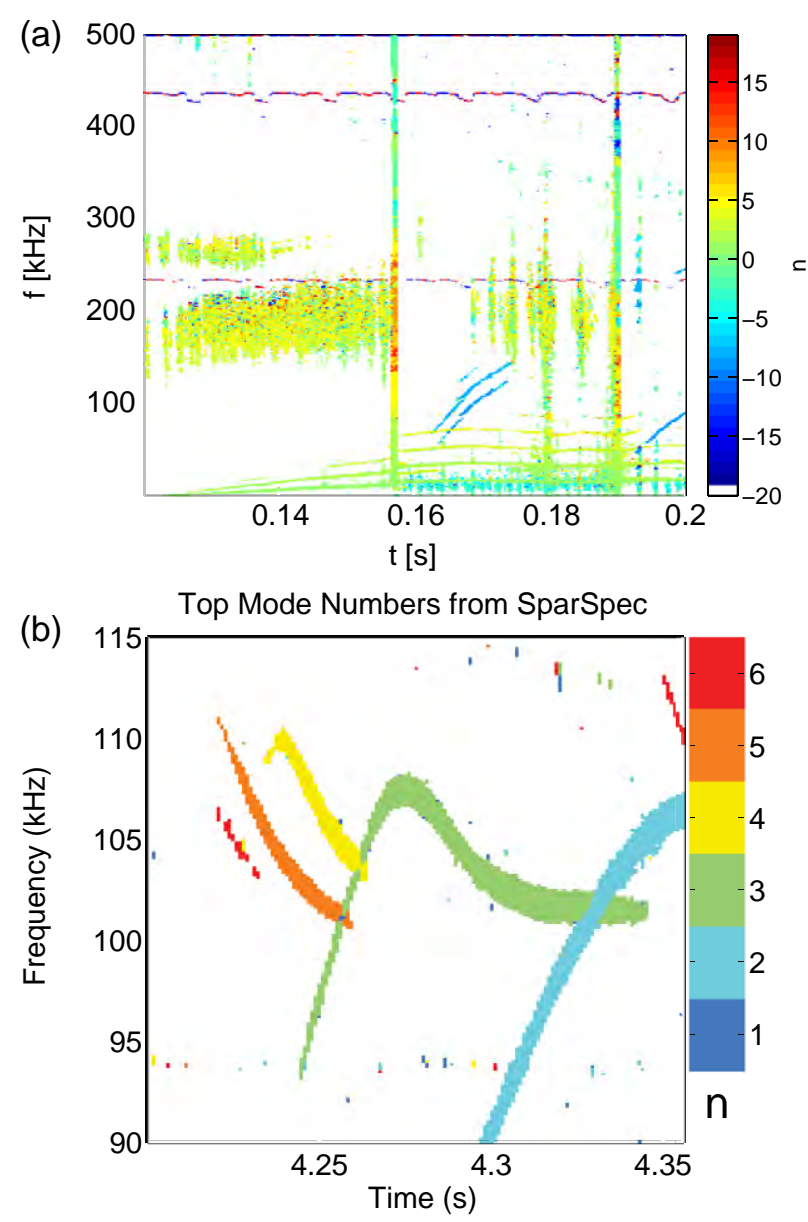

Figure 6. Toroidal mode fitting of waves in a plasma from Mirnov array data. (a) shows Fourier-SVD fit to MAST for \#9429 from 0.1 to $0.2 \mathrm{~s}$, and (b) SparSpec fit to JET shot \#69436 using eight Mirnov coils. Reproduced with permission from figure 8 of Klein et al [57].

2.2.3. Wave polarization. A three-probe set of magnetic field coils can also be used to measure wave polarization. For instance, in MAST, the OMAHA set of magnetic field probes consists of three coils wound on orthogonal axes on a common former. In Fourier space, the complex magnitude $\delta B_{R}(\omega), \delta B_{z}(\omega), \delta B_{\phi}(\omega)$ of each field coefficient is related to the voltage on the coil as follows:

$$
\begin{aligned}
\delta B_{R}(\omega) & =\frac{H_{R} V_{R}(\omega)}{j \omega N_{R} A_{R}}, \\
\delta B_{Z}(\omega) & =\frac{H_{Z} V_{Z}(\omega)}{j \omega N_{Z} A_{Z}}, \\
\delta B_{\phi}(\omega) & =\frac{H_{\phi} V_{\phi}(\omega)}{j \omega N_{\phi} A_{\phi}},
\end{aligned}
$$

where $H_{R}, H_{Z}$, and $H_{\phi}$ is the transfer function; $N_{R}, N_{Z}$ and $N_{\phi}$ the number of turns; and $A_{R}, A_{Z}$ and $A_{\Phi}$ the crosssections of each coil, respectively. The time projection of $\left(\delta B_{R}(\omega), \delta B_{z}(\omega), \delta B_{\phi}(\omega)\right)$ traces out a polarization ellipse. Characterizing the properties of the polarization, and relating these properties to that of the equilibrium field $\boldsymbol{B}$ and wave vector $\boldsymbol{k}$ requires a polarization model,

In optics and astrophysics $[59,60]$, a popular representation for the polarized electric or magnetic field vector is the 


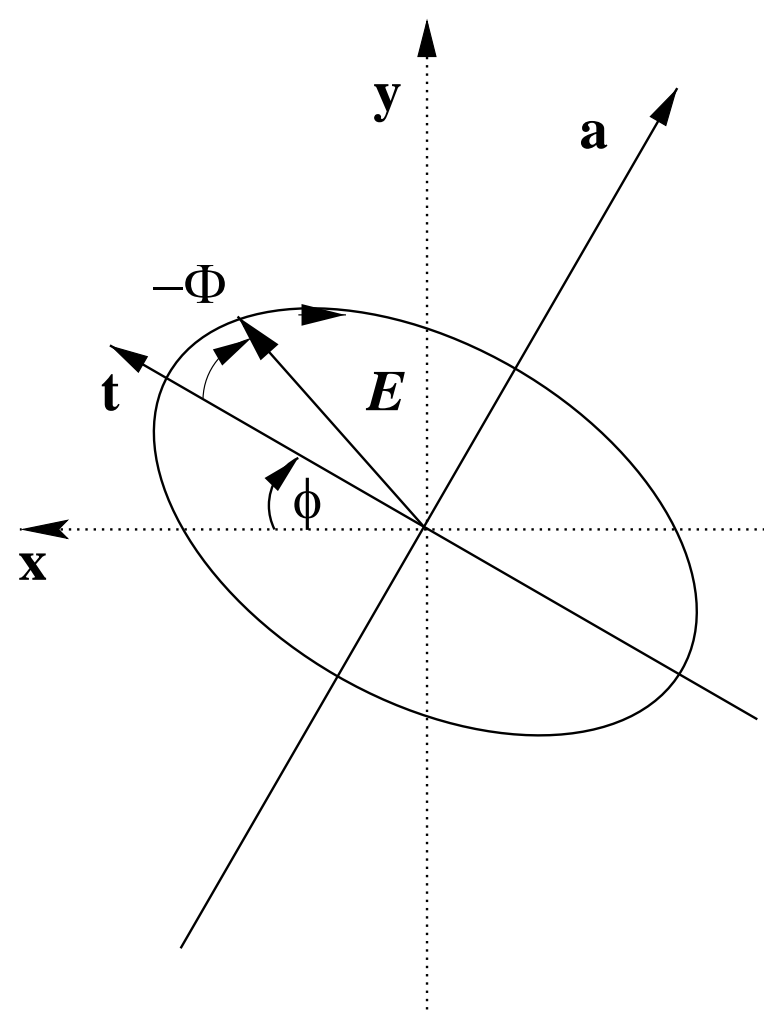

Figure 7. The rotation of an arbitrary transverse wave vector through angle $\phi$ can always be written as a polarization ellipse with $\delta B$ the peak field strength, and $\Phi$ the initial phase. The unit normal to the polarization plane, $e_{n}$, is directed into the page. Both the wave vector $\boldsymbol{k}$ and magnetic field $\boldsymbol{B}$ may be at an arbitrary angle with respect to $e_{n}$. The mode drawn here rotates in a clockwise direction, and has a negative initial phase angle.

axial-ratio formulation. In this representation, an arbitrary transverse magnetic field vector is written

$$
\delta \boldsymbol{B}=\frac{\delta B \mathrm{e}^{\mathrm{i}(\Phi-\omega t)}}{\sqrt{1+T^{2}}}\left(T \boldsymbol{e}_{t}+i \boldsymbol{e}_{a}\right),
$$

with the coordinates axes $\boldsymbol{e}_{t}$ and $\boldsymbol{e}_{a}$ taking the major and minor axis of the polarization ellipse, and $e_{n}=e_{t} \times e_{a}$ the unit normal to the polarization plane. The quantity $T$ is referred to as the axial ratio, being the ratio of the major to minor axis. As time advances, the real projections of equation (53) trace out an ellipse with axes along the $e_{t}$ and $e_{a}$ direction and initial phase $\Phi$. Figure 7 shows the coordinate geometry and the trajectory of the magnetic field vector tip for a wave with $T>0$, and negative initial phase. As time advances the wave propagates into the page, whilst the wave vector rotates in a clockwise direction, and is said to be right-hand (RH) elliptically polarized. The value of the axial ratio formulation is that if the polarization plane and major axis is known, the polarization properties of the wave are determined by one real quantity, $T$.

In order to express the perturbed magnetic field in the axial-ratio formulation, the Fourier data must be rotated to the polarization frame. In general, this can be accomplished by three frame rotations. In column vector notation, the rotated coordinate frame $\left(e_{R}^{(3)}, e_{\phi}^{(3)}, e_{Z}^{(3)}\right)$ is related to the laboratory frame $\left(e_{R}, e_{\phi}, e_{Z}\right)$ via

$$
\left(\begin{array}{c}
e_{R}^{(3)} \\
e_{\phi}^{(3)} \\
e_{Z}^{(3)}
\end{array}\right)=R_{3} \cdot R_{2} \cdot R_{1} \cdot\left(\begin{array}{c}
e_{R} \\
e_{\phi} \\
e_{Z}
\end{array}\right),
$$

where $\boldsymbol{R}_{1}, \boldsymbol{R}_{2}$ and $\boldsymbol{R}_{3}$ are rotation matrices, and where the superscript $^{(n)}$ denotes the frame rotated by $\boldsymbol{R}_{n} \cdot \ldots \cdot \boldsymbol{R}_{1}$. The first two rotation matrices $\boldsymbol{R}_{1}$ and $\boldsymbol{R}_{2}$ rotate the laboratory frame so that $\boldsymbol{e}_{R}^{(2)}=\boldsymbol{e}_{n}$. These rotations can be chosen in two arbitrary orthogonal directions, and we have selected $\chi_{1}, \chi_{2}$ to be rotations about the $e_{Z}$ and $e_{\phi}^{(1)}$ axes, respectively. The Fourier components in the $\left(e_{R}^{(2)}, e_{\phi}^{(2)}, e_{Z}^{(2)}\right)$ and $\left(e_{R}, e_{\phi}, e_{Z}\right)$ frames are then related by

$$
\begin{gathered}
\left(\begin{array}{c}
0 \\
F_{\Phi}^{(2)} \\
F_{Z}^{(3)}
\end{array}\right)=\left(\begin{array}{ccc}
\cos \left(\chi_{2}\right) & 0 & -\sin \left(\chi_{2}\right) \\
0 & 1 & 0 \\
\sin \left(\chi_{2}\right) & 0 & \cos \left(\chi_{2}\right)
\end{array}\right) \\
\cdot\left(\begin{array}{ccc}
\cos \left(\chi_{1}\right) & \sin \left(\chi_{1}\right) & 0 \\
-\sin \left(\chi_{1}\right) & \cos \left(\chi_{1}\right) & 0 \\
0 & 0 & 1
\end{array}\right) \cdot\left(\begin{array}{l}
F_{R} \\
F_{\Phi} \\
F_{Z}
\end{array}\right) .
\end{gathered}
$$

The real and imaginary components of complex equation $F_{R}^{(2)}=0$ is solved for the rotation angles $\chi_{1}$, and $\chi_{2}$. This yields

$$
\begin{gathered}
\tan \chi_{1}=\frac{F_{Z}^{i} F_{R}^{r}-F_{R}^{i} F_{Z}^{r}}{F_{Z}^{r} F_{\phi}^{i}-F_{\phi}^{r} F_{Z}^{i}}, \\
\tan \chi_{2}=\frac{F_{R}^{r} \cos \chi_{1}+F_{\phi}^{r} \sin \chi_{1}}{F_{Z}^{r}},
\end{gathered}
$$

where the superscript ${ }^{r}$ and ${ }^{i}$ denote real and imaginary components, respectively. The third rotation, $\chi_{3}$ is chosen to rotate $\left(F_{\phi}^{(2)}, F_{Z}^{(2)}\right)$ in the polarization plane onto the major and minor axis. That is,

$$
\left(\begin{array}{l}
F_{\phi}^{(3)} \\
F_{Z}^{(3)}
\end{array}\right)=|\delta B| e^{i \Phi}\left(\begin{array}{l}
T \\
i
\end{array}\right) .
$$

Equation (58) comprises two independent equations, which can be solved for the axial-ratio $T$ and the rotation angle $\chi_{3}$. This yields for $\chi_{3}$

$$
\tan \chi_{3}=\frac{T F_{Z}^{(2) i}-F_{\phi}^{(2) r}}{T F_{\phi}^{(2) i}+F_{Z}^{(2) r}},
$$

together with the quadratic

$$
\begin{gathered}
T^{2}\left[F_{Z}^{(2) r} F_{\phi}^{(2) i}-F_{Z}^{(2) i} F_{\phi}^{(2) r}\right]+T\left[\left(F_{Z}^{(2) r}\right)^{2}+\left(F_{Z}^{(2) i}\right)^{2}+\left(F_{\phi}^{(2) r}\right)^{2}\right. \\
\left.+\left(F_{\phi}^{(2) i}\right)^{2}\right]+\left[-F_{Z}^{(2) i} F_{\phi}^{(2) r}+F_{\phi}^{(2) i} F_{Z}^{(2) r}\right]=0 .
\end{gathered}
$$

The two solutions for $T$ have the same sign, and are equal to their inverse. Without loss of generality, we have chosen the solution with $T>1$, such that $e_{t}$ is the major radius.

To extract the unit vector $e_{t}, e_{a}$ and $e_{n}$ in the laboratory frame the inverse rotations are performed. For example, in the laboratory frame the polarization plane $\boldsymbol{e}_{n}^{(-3)}$ is

$$
\boldsymbol{e}_{n}^{(-3)}=\boldsymbol{R}_{1}^{-1} \cdot \boldsymbol{R}_{2}^{-1} \cdot \boldsymbol{R}_{3}^{-1}\left(\begin{array}{l}
1 \\
0 \\
0
\end{array}\right)
$$


Appel et al [58] have extracted the polarization of modes from low and high frequency MAST data, the latter of which is believed to be CAE activity. Figure 8 shows the computed value of $e_{n} \times \boldsymbol{B}$ for both low and high frequency modes in MAST discharge \#17944. At the times shown, the on-axis TAE frequency is $120 \mathrm{kHz}$, and the high frequency modes are in the frequency interval $\omega_{c i} / 2<\omega<\omega_{c i}$, with $\omega_{c i}$ the ion cyclotron frequency. Both low and high-frequency modes are elliptically polarized.

2.2.4. Wave-wave coupling. The tools above are linear to describe the frequency and mode numbers of wave oscillations. Higher order spectral tools, such as the bispectrum [61, 62], offer quantitative techniques to resolve and quantify the phase relationships between harmonics. The bispectrum is defined by

$$
B\left(\omega_{k}, \omega_{l}\right)=\left\langle X\left(\omega_{k}\right) X\left(\omega_{l}\right) X^{*}\left(\omega_{k}+\omega_{l}\right)\right\rangle,
$$

where $X$ is the Fourier transform of the signal computed over a time window $\Delta t$, and $*$ denotes the complex conjugate. The \langle\rangle brackets denote an averaging operation over a time window $\Delta \tau$, with $\Delta \tau>\Delta t$. The bispectrum, with magnitude $|B|$ and biphase $\angle B$, identifies and resolves frequency components that satisfy the frequency coupling condition $\omega_{k}+\omega_{l}=$ $\omega_{k+l}$. The properties of the bispectrum depend largely upon the phase $\angle X\left(\omega_{k}\right) X\left(\omega_{l}\right) X^{*}\left(\omega_{k}+\omega_{l}\right)$ over each window $\Delta t$. Three different types of signals produce different phase relationships [62]:

(i) For signals that are coherent over the interval $\Delta \tau$ and exhibit frequency coupling, but are not the result of nonlinear modulation, the phase $\angle X\left(\omega_{k}\right) X\left(\omega_{l}\right) X^{*}\left(\omega_{k}+\right.$ $\left.\omega_{l}\right)$ will be the same for every window $\Delta t$ within $\Delta \tau$. The biphase need not be zero. That is, in general, $\Phi_{l}+\Phi_{k}-\Phi_{l+k} \neq 0$. Such a signal might correspond to three independently driven cavity modes with frequencies $\omega_{1}, \omega_{2}=2 \omega_{1}$ and $\omega_{3}=3 \omega_{1}$.

(ii) For signals that are the result of a quadratic nonlinearity the biphase at all peaks in the bispectrum amplitude (which correspond to signals with components at frequency $\omega_{k}, \omega_{l}$ and $\omega_{k+l}=\omega_{k}+\omega_{l}$ ) is always zero. A signal of the form $x_{p}=$ $\sum_{i=1}^{M} A_{i} / 2\left[\exp \left(j\left(\omega_{i} t+\Phi_{i}\right)\right)+\exp \left(-j\left(\omega_{i} t+\Phi_{i}\right)\right)\right]$ when driven to produce a signal $y_{N L}$ of nonlinear order $N$ through

$$
y_{N L}=\sum_{\gamma=1}^{N} \beta_{\gamma} x_{p}^{\gamma}+x_{\text {noise }},
$$

where $\beta_{\gamma}$ are arbitrary real coefficients and $x_{\text {noise }}$ a noise component, will have zero biphase at all peaks in the bispectrum. That is, if $\Omega^{a}, \Omega^{b}$ and $\Omega^{a+b}$ are frequencies given by $\Omega^{a}=\sum_{i=1}^{M}\left(k_{i}^{a}-k_{M+i}^{a}\right) \omega_{i}, \Omega^{b}=\sum_{i=1}^{M}\left(k_{i}^{b}-\right.$ $\left.k_{M+i}^{b}\right) \omega_{i}$, with $k_{i} \in \mathbb{N}$, and $k_{i}, k_{i+M}$ are the power indices of the $\omega_{i}$ and $-\omega_{i}$ components respectively, and $\Omega^{a+b}=$ $\Omega^{a}+\Omega^{b}$, then we find

$$
\begin{gathered}
\angle B\left(\Omega^{a}, \Omega^{b}\right)=\sum_{i=1}^{M}\left[\left(k_{i}^{a}-k_{M+i}^{a}\right)+\left(k_{i}^{b}-k_{M+i}^{b}\right)\right. \\
\left.-\left(k_{i}^{a}+k_{i}^{b}-k_{M+i}^{a}-k_{M+i}^{b}\right)\right] \Phi_{i}=0 .
\end{gathered}
$$

Such signals satisfy the phase coupling condition $\Phi_{l}+$ $\Phi_{k}=\Phi_{l+k}$. A plasma example is a single mode driven nonlinearly to large amplitude, producing harmonics.

(iii) For frequencies $\left(\omega_{k}, \omega_{l}\right)$ which correspond to noise, the $\angle X\left(\omega_{k}\right) X\left(\omega_{l}\right) X^{*}\left(\omega_{k}+\omega_{l}\right)$ will be uniformly distributed. The averaging operation in the bispectrum has no effect on signals that are coherent, but serves to minimize the magnitude of the bispectrum value of noise, by sampling wave packets with different random biphase.

The presence of both frequency and phase coupling is very strong evidence that the signal comprises components that are the product of nonlinear modulation. A measure of signal nonlinearity often used is the bicoherence [63]:

$$
b^{2}\left(\omega_{k}, \omega_{l}\right)=\frac{\left|B\left(\omega_{k}, \omega_{l}\right)^{2}\right|}{\left\langle\left|X\left(\omega_{k}\right) X\left(\omega_{l}\right)\right|^{2}\right\rangle\left\langle\left|X\left(\omega_{k}+\omega_{l}\right)\right|^{2}\right\rangle}
$$

which lies between zero and unity. A bicoherence of zero indicates no frequency coupling at frequencies $\omega_{k}, \omega_{l}$ and $\omega_{k}+\omega_{l}$. While a bicoherence of unity indicates frequency coupling, it does not necessarily imply phase coupling, especially if the signal is coherent over the window within which the averaging of equation (62) was performed. In this instance, to better distinguish between signals (i) and (ii), either the time window $\Delta \tau$ must be increased to span multiple wave bursts, or the biphase must be inspected.

In recent years, higher order spectral techniques such as the bispectrum have found an increasing use in the study of mode coupling. Some examples in magnetic confinement include the study of the interaction of multiple neoclassical tearing modes (NTMs) [64], plasma turbulence [65], confinement transitions [66], evidence of mode saturation in a laboratory plasma [67], and identification of three wave coupling in the Earth's foreshock region [68]. Raju et al [64] study the interaction of $m / n=3 / 2,4 / 3$ and $7 / 5$ coupled modes in JET plasmas. By studying the time evolution of the bicoherence and mode amplitude, they were able to deduce that the $7 / 5$ mode is driven by the $3 / 2$ and $4 / 3$ modes, and that the strong 4/3,3/2 coupling may be responsible for the observed stabilization of the $4 / 3$ mode on the $3 / 2$ mode. Itoh et al [65] study the bicoherence of fluctuations in a test system comprising drift waves and zonal flows. They computed the response of a test mode to nonlinear interaction between a pair of modes in a turbulent plasma, and using this, compute the turbulent spectrum and bicoherence. Amongst other results, Itoh et al find the total bicoherence increases as the amplitude of the zonal flow increases. White et al [66] study the characteristics of the bicoherence calculated from $D_{\alpha}$ oscillations measured in the edge plasma of NSTX. Their main result is that the level of nonlinear coupling between low and high frequency fluctuations does not vary in the $10 \mathrm{~ms}$ prior to confinement transition. Austin et al [67], has studied electrostatic fluctuations of a vacuum arc centrifuge [69] reveal the plasma is in a stochastic growth state [70], also employs the bicoherence to confirm the electrostatic modes are nonlinearly coupled. Recently, Narita et al [68] have estimated the bicoherence in magnetic field perturbations and density in the 

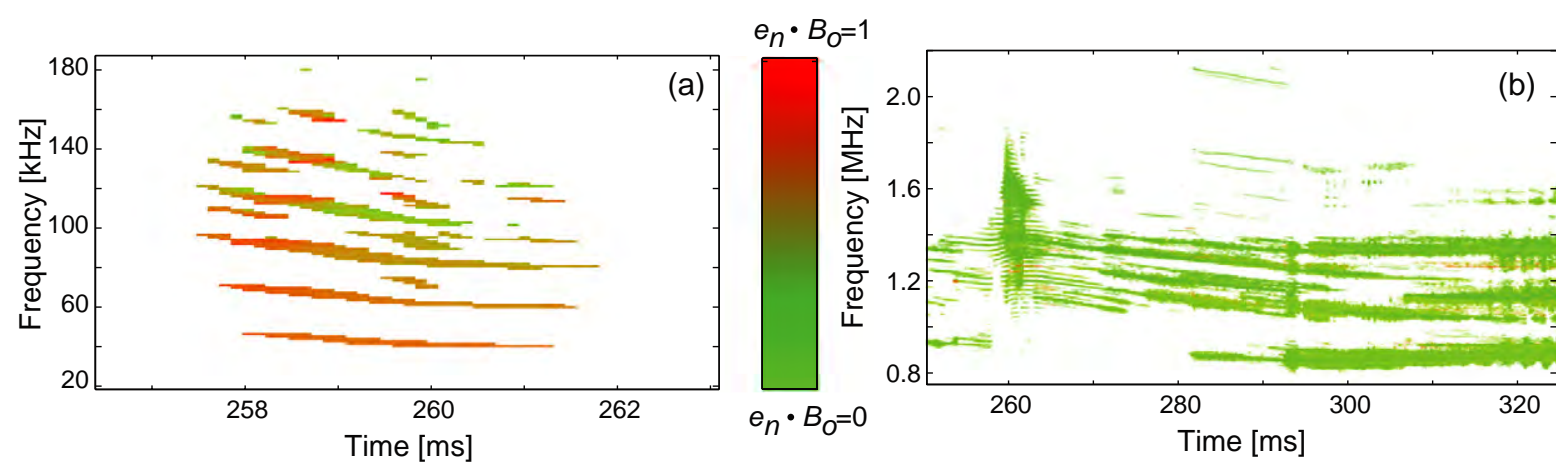

Figure 8. Orientation of the polarization plane for $(a)$ low-frequency modes and $(b)$ high- frequency modes in MAST discharge \#17944. Here, $\boldsymbol{B}_{0}$ is the equilibrium field. Reproduced with permission from figure 16 of Appel et al [58].

wavenumber domain from four Cluster spacecraft positioned in Earths foreshock region.

An example application of the bicoherence and the biphase is interpretation of the phase relationships between low and high frequency mode activity of magnetic fluctuation data from the OMAHA magnetic array in MAST [71]. In that analysis the biphase and bicoherence of low and high frequency chords of discharge \#9429 at $220 \mathrm{~ms}$ were examined, and the modes found to be consistent with quadratic phase coupling, such that $\Phi_{l}+\Phi_{k}-\Phi_{l+k}=0$ and the frequency response of the amplifier featured a cubic nonlinearity at high frequency. Figure 9 shows the biphase and bicoherence of MAST magnetics for discharge \#9429 at $220 \mathrm{~ms}$, together with the unwrapped biphase chords.

2.2.5. Wave statistics. In the last 10-15 years the calculation of wave statistics has become popular in laboratory plasmas in attempts to quantify the state of the underlying plasma oscillations. Such tools are common to astronomy and astrophysics, where data is extremely poor in spatial resolution, and therefore more extensive signal processing is applied to time series data to maximize information extraction.

A range of theories now exist to describe wave field statistics in inhomogeneous stochastic systems/media, including self-organized criticality (SOC) [72], which predicts power law distributions for the electric field strength; scattering by density irregularities [73] which produces Gaussian statistics; strong turbulence [74], which predicts power-law distributions; and stochastic growth theory [70], which predicts lognormal field distributions. Physically, each of these various theories are differentiated by the varying types of interaction between the waves and background medium.

The study of wave statistics has also become more common in laboratory plasma physics, in a quest to use different techniques to infer physics of important plasma processes. Austin et al [67] have computed the statistics of the wave envelope of electrostatic field oscillations in a plasma centrifuge. They have found that the wave envelope demonstrated lognormal distribution functions, and therefore was consistent with the description of stochastic growth theory for which the mean plasma growth rate was at marginal stability.

By computing the pdf of the ELM inter-burst interval, Greenhough et al [75] have shown that inter-burst-interval
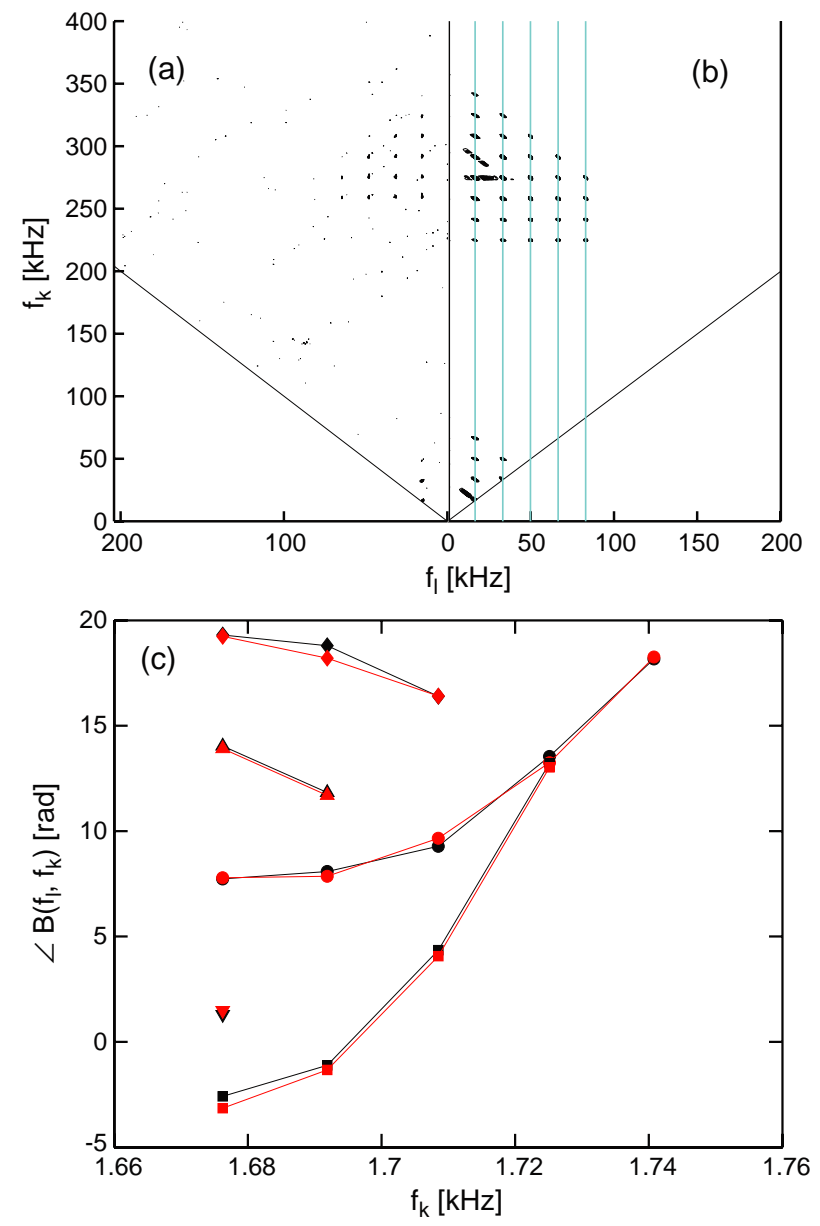

Figure 9. Biphase and bicoherence of MAST magnetics for discharge \#9429 at $220 \mathrm{~ms}$. Figure $(a)$ and $(b)$ show the measured and predicted bicoherence, and figure $(c)$ shows the unwrapped biphase chords, showing measured (heavy) and predicted (light) biphase. Reproduced with permission from figures 8 and 10 of Hole and Appel [71].

pdf's can be either Gaussian or inverse exponential ${ }^{1}$; and it is possible to distinguish inverse exponential cases that do, or do not, reflect a Poisson process. This suggests that there may be several fundamentally different kinds of underlying nonlinear processes at work. Much more recently, Webster and

1 An inverse exponential function is one of the form $f(x)=\eta / x^{2} \exp (-\eta / x)$, with $\eta$ a scale length. 
Dendy [76] have established the waiting times ELMs should obey a Weibull distribution, providing that the conditional probability that another ELM follow the immediate previous ELM obeys a power law distribution. They show this for JET type I and type III ELMs.

In other work, Chapman and co-workers [77] have shown that confinement phenomenology characteristic of magnetically confined plasmas emerges naturally from a simple sandpile algorithm when the parameter controlling redistribution scale length is varied. Building on this work, Rhee et al [78] recently constructed an extended cellular automata model that reproduces key qualitative features of ELM mitigation experiments, most significantly the increase in frequency of grain ejection events (ELMs), and the decrease in the number of grains ejected by these transport events.

Statistical analysis techniques have also been used to analyse the structure and intermittency of localized plasma 'blobs' or coherent structures (see D'Ippolito et al [79] and references therein), whose formation is often linked to wavelike interchange or drift oscillations in basic plasma devices, or in fusion plasmas, turbulence or ELMs. A pronounced feature across many magnetic geometries (see Garcia et al [80] and references therein) is a skewness and flattening of the pdf of particle density and temperature in the plasma edge region, consistent with the interpretation of coherent or blob-like structures. Statistical characterization also suggests computational techniques to model blob transport, such as the replacement of blobs with 'macro-blobs' in transport codes such as UEDGE [81]. The replacement is valid providing the statistics of plasma perturbations are identical using blob and macro-blob models.

\subsection{Particles}

2.3.1. Simulation. Simulation is the most wide spread used tool to diagnose fast particle populations toroidal magnetic confinement. Monte Carlo beam codes such as the NUEBEAM module [82] of TRANSP [12] contain a Monte Carlo package for time dependent modelling of fast ion species in an axisymmetric tokamak. This Monte Carlo package represents the fast ion slowing down distribution function as a discreet set of weighted model ions. Gyrophase averaged particle trajectories are followed down to thermal energies: NUBEAM stops following ions that slow down below $(3 / 2) T_{\mathrm{i}}$, where $T_{\mathrm{i}}$ is the temperature of the thermal ions. These ions are then considered as thermalized ions and are described in terms of a thermalization source function provided as an output of the NUBEAM module.

A wider number of test particle full-orbit codes exist, such as CUEBIT, [83] which are useful for physics exploration, but do not have a self-consistent electric field. Such codes have been generalized to include the collisional interaction of suprathermal test-particles with a Maxwellian population of field particles in the presence of toroidal magnetic field ripple [84]. Figure 10 shows the projections onto the poloidal plane of the collisionless passing (those that are not toroidally trapped) particle orbits of beam ions that are (a) well-confined and (b) marginally-confined. In MAST, the beam ion deposition
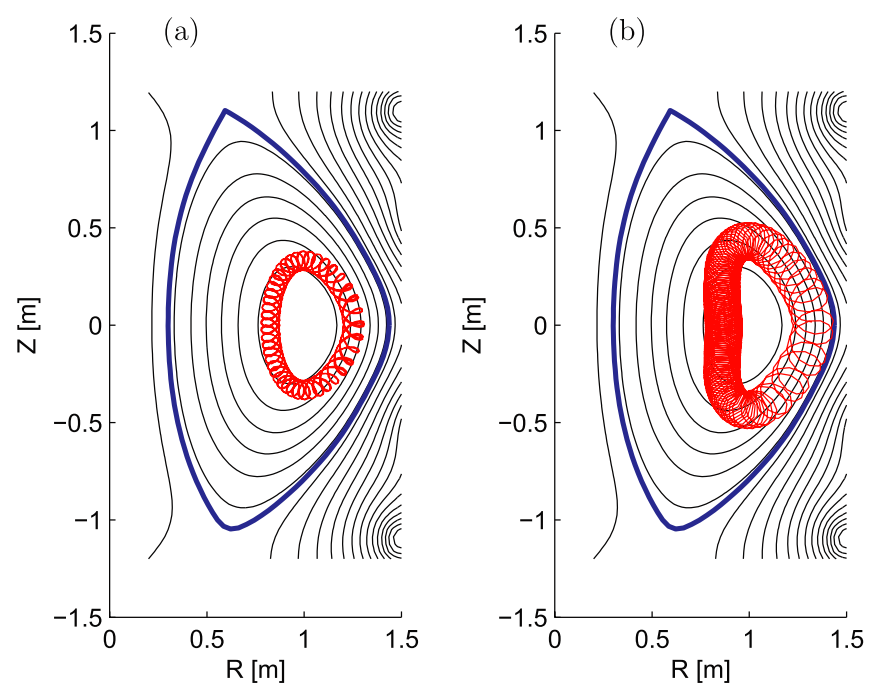

Figure 10. Projections onto the poloidal plane of collisionless beam ion orbits in MAST. The ions were born at $(a) R=0.81 \mathrm{~m}$, $Z=0.12 \mathrm{~m}$ and $(b) R=1.32 \mathrm{~m}, Z=0.02 \mathrm{~m}$. Black curves show flux surface contours, with the last closed flux surface in blue. Reproduced with permission from figure 2 of [84]. Copyright 2012 AIP Publishing LLC.

profile extends up to the plasma edge, and the majority of beam atoms are ionized well before they reach the tangency radius. Thus, the impact of toroidal field ripple will be large, and as the Larmor radius is large owing to the relatively weak toroidal field, full orbit effects may be important. McClements et al find that the effect of toroidal field ripple accounts for only a small contribution to anomalous transport rates that have been invoked to account for measured neutron rates and plasma stored energies in some MAST discharges.

2.3.2. Particle measurements. Neutral particle analysers (NPA) measure the energy spectra of neutral particles collected along a line of sight of a viewing chord, (see Tournianski et al [85] and references therein) and form the main diagnostic on many machines to study the confinement of fast ions. Neutral particles travelling along the line of sight of the viewing chord are predominantly either injected neutrals that undergo Coulomb scattering with an impurity ion in the plasma, or are produced by charge exchange with a confined ion. The energy spectra extends up to the injection energy of neutral beams. A complete interpretation of NPA spectra is critically dependent on Monte Carlo particle simulations such as TRANSP, which simulate the neutral particle flux. The degree of agreement with the simulated and measured NPA spectra provides evidence of whether transport processes are correctly captured, and the level of anisotropy.

A direct measure of the slowing down of deuterium ions is available through fast ion D-alpha (FIDA) diagnostic. In this challenging diagnostic, energetic deuterium ions undergo charge exchange with a beam or edge neutral alpha, and the neutral emits $\mathrm{D}_{\alpha}$ light. The $\mathrm{D}_{\alpha}$ line is Doppler shifted away from other bright interfering signals. Analysis yields the fast-ion velocity distribution function and spatial profile. [86] Since developed in 2007, FIDA has now been deployed on NSTX, DIII-D, ASDEX Upgrade, TEXTOR, MAST 
and LHD. FIDA has been useful in contributing to the understanding of redistribution of fast ions. For instance, FIDA measurements of the fast-ion populations in MAST reveal strong redistribution of fast ions in both real and velocity space as a result of fishbones [87], confirming that anomalous (higher than neoclassical) fast-ion redistribution in MAST appears to be due mainly to MHD.

Fast ions that lose confinement can be directly measured. A scintillator based fast ion loss detector (FILD) acts as a magnetic spectrometer, dispersing fast-ions onto a scintillator, with the strike points depending on their gyro-radius energy and pitch angle between ion velocity and magnetic field line [88]. The short decay time of some scintillators (e.g. $490 \mathrm{~ns}$ for TG-Green scintillator, Sarnoff Corporation, USA) means that they can be used in conjunction with wave diagnostics, such as Mirnov coils, to study wave induced fast ion losses. For example, figure 11 shows the correlation between magnetic and FILD spectrograms in AUG [89]. Synthetic diagnostic codes have been written to simulate the scintillator measurements in quiescent [90] and MHD-rich [91] plasmas. Finally, neutrons from fusion reaction can be detected by either scintillators or by an array of indirect measurements such as a fission chamber. Together with other diagnostics these are often used to characterize the impact of particle redistribution during MHD (e.g. [91]).

\section{Wave-particle interaction}

We begin by a brief review of the physics of the wave-particle resonant interaction with an example of linear resonant drive of a TAE, inclusion of some damping physics (continuum), and calculation of the nonlinear evolution of wave amplitude and resonant part of the distribution function. We furnish our calculations with some relevant developments in wave-particle interaction physics.

\subsection{Linear drive}

Alfvén waves are driven unstable by the free energy of the energetic particle distribution function. Power transfer between a particle population with particle velocity distribution function $f_{0}$, the resonant part of the distribution function $f$, and a wave field producing Lorentz force $\boldsymbol{F}=e Z(\boldsymbol{E}+\boldsymbol{v} \times \boldsymbol{B})$, is

$$
P_{\alpha}=\frac{\mathrm{d}}{\mathrm{d} t} \iint(\boldsymbol{F} \cdot \mathrm{d} \boldsymbol{l}) f \mathrm{~d} x^{3} \mathrm{~d} v^{3},
$$

with $v=\mathrm{d} l / \mathrm{d} t$ the velocity vector. Expanding in cylindrical coordinates $r, \vartheta$, $\phi$, equation (66) becomes

$$
P_{\alpha}=\int \mathrm{d} \varphi \mathrm{d} \theta \mathrm{d} r R \mathrm{~d} r \int \mathrm{d} v f\left(-e \boldsymbol{v}_{d} \cdot \boldsymbol{E}_{\perp}\right),
$$

where for shear Alfvén waves $E_{\|}=0$, and $\boldsymbol{v}_{d}$ is the guiding centre drift velocity. Using the mode representation $\boldsymbol{E}=\nabla \phi_{m} \exp (i(n \varphi-m \theta-\omega t))$ with $E_{\|}=0$ of equation (3) of Berk et al [92] a linearized kinetic equation for the function
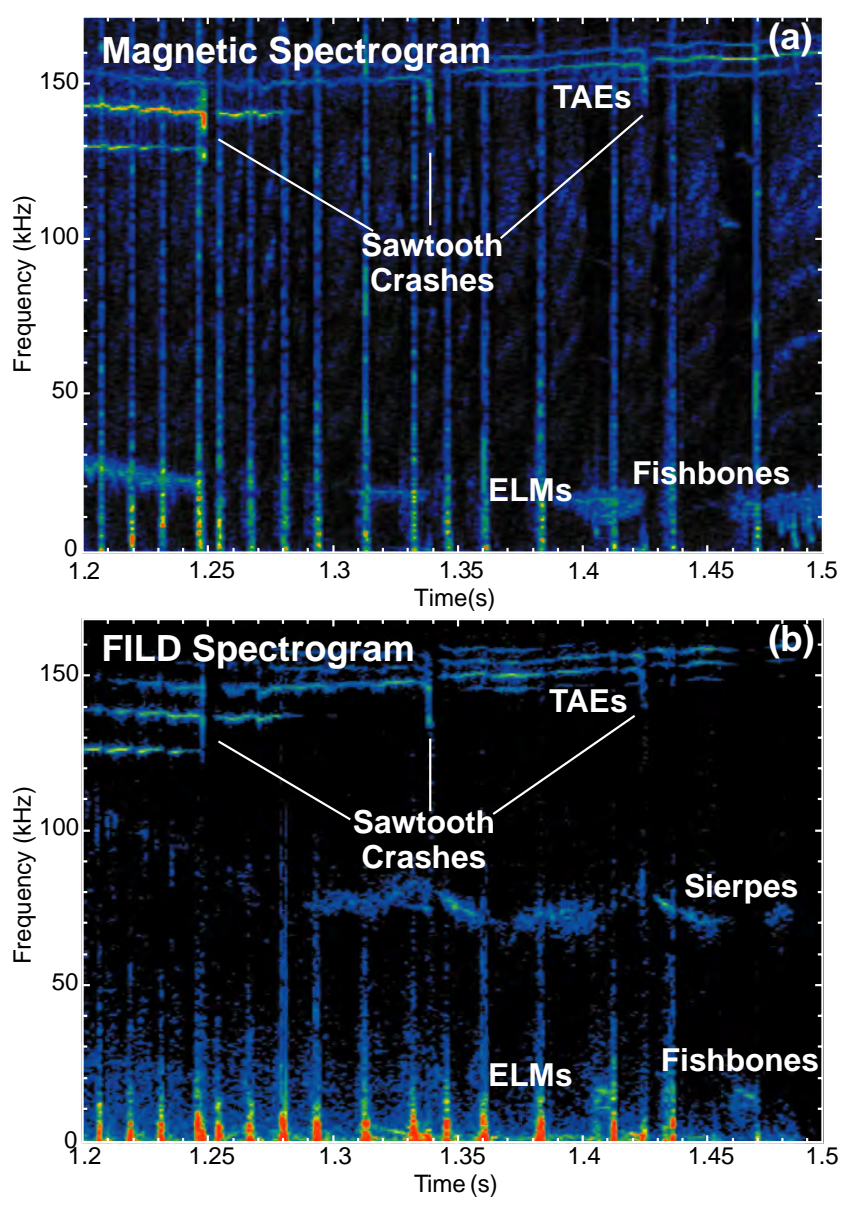

Figure 11. AUG discharge \#21011: (a) spectrogram of a magnetic pick-up coil signal, $(b)$ spectrogram of a FILD channel. Reproduced with permission from figure 9 of [89]. Copyright 2009 AIP Publishing LLC.

$f$ can be written, and the condition for resonant energetic particles identified as

$$
\omega+(m+l) \dot{\theta}-n \dot{\varphi}=0,
$$

where the wave potential has been expanded as a Fourier series:

$$
\phi_{m}=\sum_{l=0}^{\infty} \phi_{m, l} \cos (l \theta),
$$

Only particles with orbits satisfying the resonance condition contribute to nonzero $P_{\alpha}$.

In the absence of collisions, particle motion of an ion is well described by the invariance of motion, particle energy $W=m v^{2} / 2$, the first adiabatic invariant of magnetic moment $\mu=m v_{\perp}^{2} /(2 B)$, and the toroidal canonical momentum $P_{\zeta}=m v_{\zeta} R-\psi$, where $R$ is the guiding centre of the particle. Particle orbits can be classified according to these constants of motion. Figure 12 shows an example from Heidbrink [93], illustrating classes of particle orbits. Passing and counterpassing refer to the direction of the toroidal magnetic field, the lost region describes particles lost to the wall, and trapped describes particles that are toroidally trapped.

As an illustration of linear wave drive, Appel and Hole [94] computed TAE drive for a MAST discharge using both 


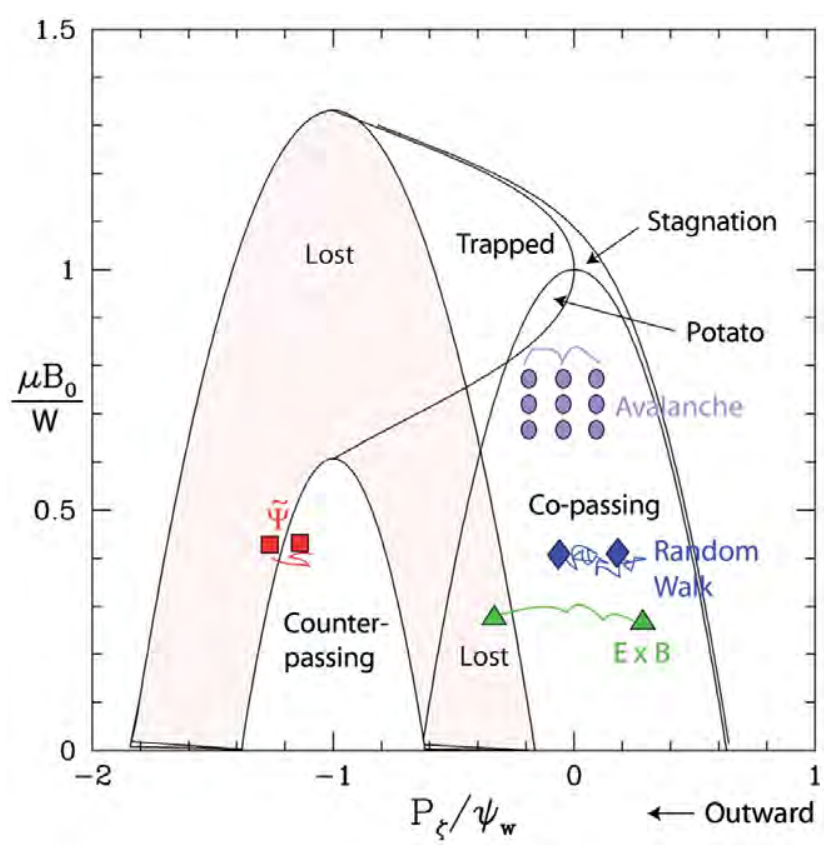

Figure 12. Classification of different orbit types for beam ions in the DIII-D tokamak versus $\mu$ and $P_{\zeta}$ The poloidal flux at the wall is $\Psi_{w}$, the particle energy is $W$, and the magnetic field at the magnetic axis is $B_{0}$. Particles that move outwards from the magnetic axis move leftwards on the $P_{\zeta}$ axis. Four types of energetic particle transport are illustrated. (Red square) The wave can perturb the equilibrium, causing particles near a loss boundary to collide with the wall. (Green triangle) Particles that stay in phase with a mode throughout the plasma can convectively escape via the $\boldsymbol{E} \times \boldsymbol{B} \mathrm{drift}$ (Blue diamond) Particles can diffuse as they receive velocity kicks associated with the many wave-particle resonances in the plasma. (Purple circles) If the energetic particles move outwards, they can locally alter the energetic particle gradient and destabilize a new wave that transports them further, where a new wave is destabilized. Reproduced with permission from figure 10 of [93]. Copyright 2008 AIP Publishing LLC.

expressions $P_{\alpha}$ and the wave particle interaction code HAGIS [95]. Using the resonance condition $v_{\|}=v_{A} /(1-2 l)$, they projected the distribution function on the resonant plane $v_{\|}=\sqrt{(E-\mu B) / M}$ for co-passing and counter-passing particles and computed the derivative $\partial f_{0} /\left.\partial p_{\zeta}^{0}\right|_{\mu, v_{\|}}$appearing in equation (67). Excellent agreement was found between HAGIS and analytic working in the linear growth rate of co and counter-passing populations.

Multiple resonances can also be driven simultaneously by the same population of particles. Schneller et al [96] treat the interaction of a $55 \mathrm{kHz}$ reverse shear Alfvén eigenmode and a $120 \mathrm{kHz}$ TAE. This treatment examines the resonance of modes in the same phase-space area using the wave particle interaction code HAGIS. The analysis found the striking result that depending on the radial mode distance, doubleresonance is able to enhance growth rates as well as mode amplitudes, without a radial mode overlap of the modes [97]. In Hole et al [98], a global and core TAE was found at the same frequency for an anisotropy-modified MAST discharge: a double-resonant wave-particle interaction between theses modes suggests the possibility of significant growth rate modification.
The calculation of linear growth rates from approximations or slices of the distribution function is widespread across wave-phenomena in fusion: the excitation of whistler waves by runaway electron beams in tokamaks [99-101], the drive of ion cyclotron instabilities [102], as well as EPMs [103].

\subsection{Quasilinear growth and nonlinear analysis}

Beyond the linear phase of the instability, the dynamics of the resonant interaction between the electrons and waves can be described by quasilinear theory [99, 104]. The distribution function of the particles is then separated into a slowly and a rapidly varying part, and only the average effect of the rapidly varying part on the slowly varying part is taken into account. The slowly varying part of the distribution is then described by a diffusion equation in phase space, and the rate of growth of wave-energy is equal to the linear growth rate. Such approaches have been used extensively to model the evolution of the distribution function up to the onset of saturation. The dynamics of the resonant interaction between particles and waves can be described by quasi-linear theory if the following conditions are satisfied: (i) the waves should be incoherent and have a sufficiently dense spectrum, in order to avoid particle trapping, (ii) the wave amplitudes should be small enough to affect the zero-order particle orbits and distribution functions to an extent negligible for the linear theory, and (iii) damping and growth rates of the waves should be small with respect to their real frequencies [105]. Such approaches have been used extensively to model the evolution of the distribution function up to the onset of the saturation regime [106].

The long term nonlinear evolution of the wave and resonant particle population for a fixed equilibrium configuration can be advanced by either long time simulation in HAGIS, or one dimensional (1D) line resonance model developed by Berk et al [107], its numerical implementation (the Bump-on-Tail code) and extensions [108,109]. The 1D line resonance model couples to the magnetic configuration and particle distribution function weakly. The wave frequency, electrostatic eigenfunction, and magnetic configuration do not appear in the 1D line resonance model. The resonant distribution function contributes only through a linear growth rate $\gamma_{l}$, and damping with the background through a single term $\gamma_{d}$. These codes are electrostatic, and do not have an electromagnetic field. Comparisons to experiments are afforded through the width of the line resonance and the constants reflecting velocity space diffusion, dynamical friction and Krook collisions. Despite these limitations, a qualitative match can be obtained between the nonlinearly frequency sweeping activity and 1D simulations. Figure 13 shows chirping hole and clump formation in MAST, detailed HAGIS modelling results, and bump-on-tail simulations. The chirping modes correspond to hole-clumps in distribution space, in which an initially resonant wave at frequency $\omega / k$ causes a local change in the distribution function $+\Delta f$ and $-\Delta f$ above and below the resonant phase speed. HAGIS reproduces the theoretically expected chirp frequency shift, as well as saturation of the chirp. In the bump-on-tail simulation, multiple bursts are generated by particle conservation. Since 

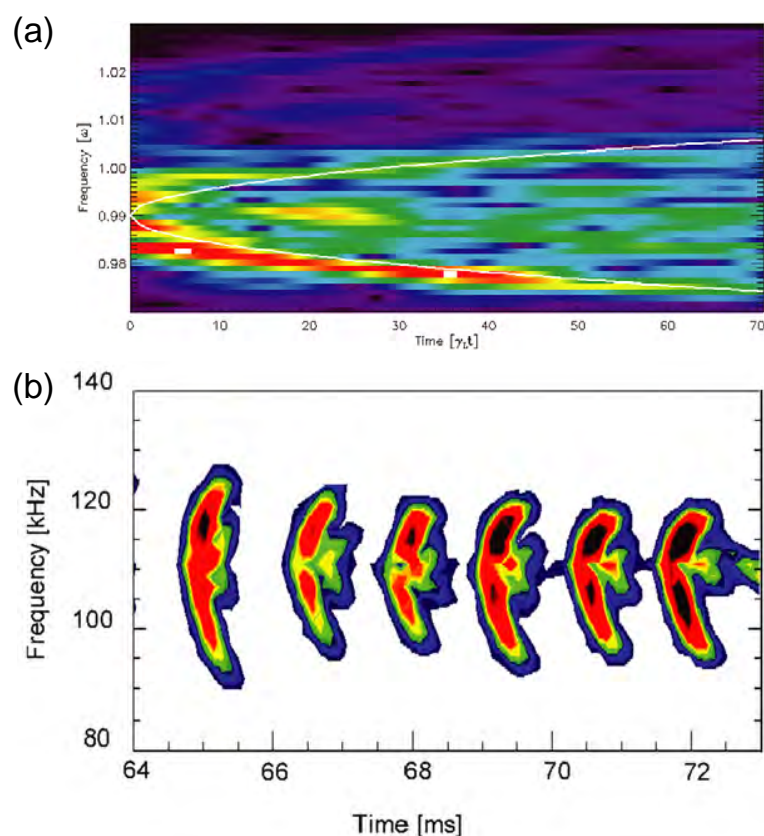

(c)

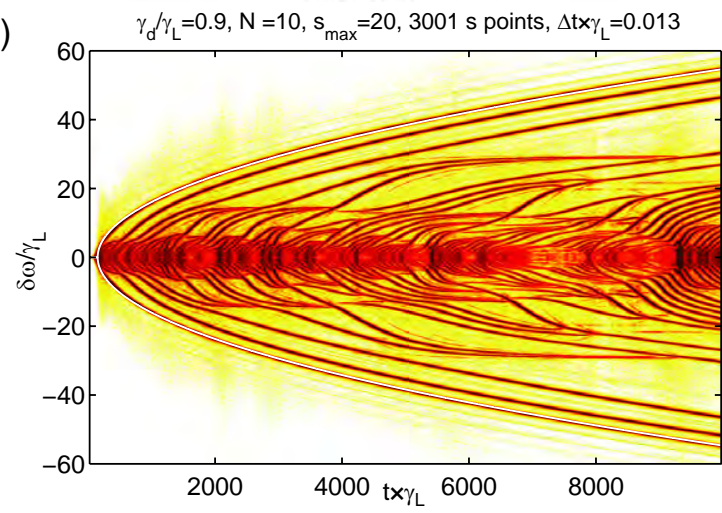

Figure 13. (a) Magnetic spectrogram showing frequency sweeping $n=1$ core-localized mode in MAST \#5568. The first event shows a frequency shift of $18 \mathrm{kHz}$ in a time of $0.8 \mathrm{~ms}$. Reproduced from figure 9 of [112]. (b) Sliding Fourier spectrum showing frequency evolution of marginally unstable TAE mode in response to kinetic $\alpha$ particle drive $\gamma_{L} / \omega_{0}=4.5 \times 10^{-3}$ and external damping $\left(\gamma_{d} / \omega_{0}=4 \times 10^{-3}\right)$. The over-plotted white line shows the theoretically predicted frequency shift of $0.4 \gamma_{L}\left(\gamma_{d} t\right)^{1 / 2}$. Reproduced from figure 8 of [112]. (c) Spectrogram of the electric field amplitude $E_{1}$ for the collisionless case close to the threshold. Reproduced with permission from figure 7 of [109]. Copyright 2010 AIP Publishing LLC.

the particle number is conserved, a number of particles must be displaced during the motion of a hole or a clump, which leads to a slight excess behind a hole and a depletion behind a clump. The resulting steepening of gradient in the distribution function makes the system susceptible to instability once again. It is expected long-time simulation will flatten the distribution function, leading to a global plateau that determines the maximum amount of energy that can released from the system for the prescribed modes.

The Berk and Breizman model has also provided a development environment to enable calculation of isolated phase-space structures, such as a hole-clump pair. Lesur and Diamond [110] explore the relationship between the wave energy density and the phasestrophy, $\Psi=\int_{- \text {inf }}^{\text {inf }}\left\langle\delta f^{2}\right\rangle \mathrm{d} v$, where the angle brackets denote the spatial average. They present a general relation between wave energy and phasestrophy, compare phasestrophy and wave energy density growth of a single phase-space structure, and obtain a simple expression for the growth rate of a single phase-space structure in the collisionless limit. By updating the width of the resonant velocity distribution function, they find reasonable agreement between the collisionless phase-space structure growth rate and simulation parameters, beyond the linear initial phase near marginal stability. The impact of turbulence on the phasespace dynamics of a 2D (spatial) phase space density-hole has also been addressed. Kosuga and Diamond [111] find that the impact of turbulence driven flows is to alter such drift-hole structures by shifting the drift-hole potential radially, and that the drift-holes and turbulence driven flows are dynamically coupled and can self-regulate each other.

\subsection{Wave-particle-plasma interaction}

Simulation codes such as TRANSP [12] evolve the plasma equilibrium on time scales of resistive diffusion. Rapid changes in the particle distribution function due to waveparticle drive occur on a much faster time scale, and are normally termed 'anomalous transport'. Some consequences of anomalous transport are well studied, such as fast ion loss due to MHD. Fast ions loss to the first wall is a concern for ITER, owing to the potential power loading. A recent ITPA research project [113] concluded that for ITER, the loss power fraction of NBI ions due to the 3D effect of ferromagnetic materials and ELM mitigation coils was about $0.2 \%$, rising to $0.3 \%$ in the presence of Alfvénic MHD activity and $4-5 \%$ with the ELM mitigation field. An emerging topic is wave-particleplasma interaction, in which a redistribution of particles in phase space is sufficient to cause a change in the plasma equilibrium.

Farengo et al [114] compute the alpha particle redistribution due to internal kink modes, in which the magnitude of the redistribution of particles is quantified by a diffusion coefficient. They found that at the relatively low mode frequencies expected in large devices the effect of the perturbed fields will be larger at lower energies, with a diffusion coefficient of alphas resonant with the $(1,1)$ flux surface of order $10^{-8} \mathrm{~m}^{2} \mathrm{~s}^{-1}$. One extreme way to scope the potential impact is to identify the energetic particle contribution to the plasma from beam injection, and thus provide a worst case estimate if all the particles are redistributed. In existing machines the contribution of energetic particles will be largest in spherical tokamaks, where the highest toroidal beta has been found [115]. Hole et al [116] showed that in the limit of a $100 \%$ fast particle fraction the impact of anisotropy of $p_{\perp} / p_{\|} \approx 1.7$ in a $700 \mathrm{kA}$ MAST discharge with $1.9 \mathrm{MW}$ of neutral beam heating was to lift the central safety factor by $15 \%$. In contrast, the change produced by toroidal rotation induced by the beam is small, with a reduction in central safety factor of $\approx 5 \%$. Subsequent wave mode calculations [98] showed that the impact of this change in the $q$ profile was to create both core and global TAEs at nearly the same frequency. 
An intriguing possibility associated with this new phenomena is the possibility of mode coupling between the core and edge, which may contribute to enhanced losses. Unlike the treatment of Schneller et al [96], such modes are at the same frequency.

Energetic particles can also stabilize modes. For instance, Testa and Albergante [28] show that fusion-born alphas that are not yet thermalized can stabilize ITG turbulence, thereby reducing an energy loss channel for thermal ions. In ITER at high energy gain $Q$, fusion born alphas are expected to thermalize on the electrons. Through energy equipartition, the electrons then heat the thermal ions.

The opposite phenomena is $\alpha$ channelling, in which the $\alpha$ particle energy is redirected to waves, which then transfers it rapidly to fuel ions such that the electrons are not heated and the fusion ash can be extracted [117]. Channelling 75\% of the alpha particle energy to ions can result in more than a factor of two increase in fusion reactivity [118]. Initial work focused on lower hybrid waves, but subsequent work has shown the phenomena can be simulated using neutral beams in replacement of alphas, driving Alfvén waves. More recently, Ido et al report observations [119] of a new energy channel from energetic particles to bulk ions through the geodesic acoustic mode proposed by Sasaki et al [120], thereby broadening the concept further. All are examples of engineering the slowing-down of energetic ions to maximize performance by either indirectly or directly shaping the plasma.

\section{Conclusions and future directions}

The purpose of this work was to review established and emerging techniques in diagnosing and modelling the waveparticle-plasma interaction. In today's machines, a motivation for this physics is neutral beam injection, which can drive both rotation and anisotropy. A new numerical implementation of a double-adiabatic model reveal that the impact of anisotropy can be significant, particularly in spherical tokamaks. While a basic understanding of the impact of anisotropy and flow on the magnetic configuration is well established, the detailed impact of beam parameters such as injection geometry relative to the field, tangency radius, and beam direction (co- or counterinjection) on the magnetic configuration is not well understood. The impact of anisotropy on plasma wave modes and global stability is also a rich but largely untouched topic.

Probabilistic methods such as Bayesian analysis, which integrate multiple different equilibrium diagnostics, propagate experimental error correctly. Although the last decade has seen a slow increase in the reporting of experimental errors, the correct propagation of uncertainty is still rare when computing experimentally derived properties of two or more measurements, such as thermal pressure. Instead, it is often assumed that the quantities are independent and Gaussian. A challenge for the field is to utilize available information to propagate uncertainty correctly, and/or to provide greater transparency in uncertainty calculation. Bayesian analysis also offers the opportunity to perform validation between different physical models, such as force balance, and identify faulty diagnostics in addition to current tomography. With a prescribed equilibrium model it is also now possible to infer poorly diagnosed internal profiles, such as energetic particle pressure. The use of such techniques seems promising for future experiments such as ITER with a limited diagnostic set. Indeed, it is likely DEMO will have a very restrictive diagnostic set due to higher levels of neutron and gamma fluxes, and fluences, nuclear heating and possible restricted access [121].

Significant progress has been made in wave mode characterization, spanning both high frequency diagnostic optimization, higher order spectral techniques to explore chirping modes, and mode analysis techniques. It is now routine to extract multiple mode numbers from Mirnov arrays, use bispectral techniques to examine mode coupling and phase relationships, and by analysing the statistics of events, infer properties about the underlying state of the plasma. Further opportunity exists to study the linear and nonlinear relationships between perturbed wave quantities, and thus validate or disprove theoretical models. Using the language of Bayesian statistics, the predicted relationship between perturbed wave quantities can be regarded as a forward model. In fusion, the comparison of the stochastic properties (such as the probability distribution function) between different perturbed quantities (such as the density in terms of the field) is in its infancy. These tools enable characterization of the degree of non-linearity in the system.

An increased uptake of short-decay time scintillators and fast ion loss detectors has provided quantitative comparison to full particle orbit codes, and enabled experimental verification of anomalous and/or resonant magnetic field perturbation transport. Linear, quasi-linear and nonlinear wave-particle simulations determine mode growth and saturation are routinely used to determine wave amplitude, and determine the impact of modes on particle orbits.

An emerging topic, which will be enabled in the long term by self-consistent nonlinear simulations, is the study of wave-particle-plasma interaction, in which a redistribution of confined particles in phase-space is sufficient to cause a change in the plasma equilibrium. To date, only scoping studies have been conducted to establish the possible magnitude of the effect, such as assuming the plasma is entirely a slowing down function from neutral beam injection (no thermals). Long term challenges include determining the impact from multiple-mode resonances, particularly in cases where the same resonant population can drive modes with different radial mode localizations, and thereby enhance radial transport. Such calculations will likely offer a nonlinear integrated modelling challenge, in which the equilibrium is recomputed from changes in the distribution function, and the modes recomputed.

An important issue we have not addressed at all in the review is the impact of toroidal asymmetric fields, or so-called fully $3 \mathrm{D}$ fields, on the notionally toroidally symmetric plasma configuration and plasma wave modes. In toroidally axissymmetric systems closed magnetic field lines always lies in nested magnetic flux surfaces. However, the breaking of axisymmetry in 2D tokamaks has profound implications on the structure of magnetic field lines, as the field can now support nested flux surfaces, magnetic islands, and chaotic field regions. Fully 3D structure can be spontaneously 
induced, such as magnetic islands or other helical structures such as long-lived modes, or imposed structure such as toroidal field ripple or resonant magnetic field perturbation coils, which have been experimentally shown to reduce and indeed quench ELMs. Through stellarator research, a large body of literature is devoted to understanding the magnetic configuration and wave modes in fully 3D nested plasmas. A grand open challenge is to understand the impact of magnetic islands and chaotic fields on the plasma configuration, particle confinement, wave modes and global stability.

\section{Acknowledgments}

This work was funded by the Australian Government through Australian Research Council grant FT0991899. The authors would like to acknowledge useful discussion with Segei Sharapov (CCFE), Alan Costley (CCFE), George Vayakis (ITER).

\section{References}

[1] Breizman B N and Sharapov S E 2011 Major minority: energetic particles in fusion plasmas Plasma Phys. Control. Fusion 53054001

[2] Pinches S D et al and JET-EFDA Contributors 2004 The role of energetic particles in fusion plasmas Plasma Phys. Control. Fusion 46 B187-200

[3] Holties H A, Fasoli A, Goedbloed J P, Huysmans G T A and Kerner W 1997 Determination of local tokamak parameters by magnetohydrodynamic spectroscopy Phys. Plasmas 4 709-19

[4] D'Haeseleer W D, Hitchon W N G, Callen J D and Shohet J L 1991 Flux Coordinates and Magnetic Field Structure (Berlin: Springer)

[5] Goldberger M L, Chew G F and Low F E 1956 The Boltzmann equation and the one-fluid hydromagnetic equations in the absence of particle collisions Proc. R. Soc. London 236 112-8

[6] Iacono R, Bonderson A, Troyon F and Gruber R 1990 Axisymmetric toroidal equilibrium with flow and anisotropic pressure Phys. Fluids B 2 1794-803

[7] McClements K G and Hole M J 2010 On steady poloidal and toroidal flows in tokamak plasmas Phys. Plasmas 17082509

[8] Grad H 1967 Toroidal containment of a plasma Phys. Fluids 10 137-54

[9] Grad H 1966 The guiding centre plasma Magneto-fluid and Plasma Dynamics (Proceedings of Symposia in Applied Mathematics vol 18) (New York: American Mathematical Society) pp 162-247

[10] Cooper W A, Bateman G, Nelson D B and Kammash T 1980 Beam-induced tensor pressure tokamak equilibria $\mathrm{Nucl}$. Fusion 20 985-92

[11] Pustovitov V D 2010 Anisotropic pressure effects on plasma equilibrium in toroidal systems Plasma Phys. Control. Fusion 52065001

[12] Hawryluk R J 1980 An empirical approach to plasma transport Physics of Plasmas Close to Thermonuclear Conditions vol 1, ed B Coppi (Brussels: CEC) pp 19-46

[13] Fitzgerald M, Appel L C and Hole M J 2013 EFIT tokamak equilibria with toroidal flow and anisotropic pressure using the two-temperature guiding-centre plasma Nucl. Fusion 53113040

[14] Cottrell G A 1990 Maximum entropy and plasma physics Maximum Entropy in Action (Oxford: Oxford Science)
[15] Fischer R, Dinklage A and Pasch E 2003 Bayesian modelling of fusion diagnostics Plasma Phys. Control. Fusion 45 1095-111

[16] Svensson J, Dinklage A, Geiger J, Werner A and Fischer R 2004 Integrating diagnostic data analysis for W7-AS using Bayesian graphical models Rev. Sci. Instrum. 75 4219-21

[17] Oliver Ford 2010 Tokamak plasma analysis through Bayesian diagnostic modelling PhD Thesis Imperial College, London

[18] Hole M J, von Nessi G, Bertram J, Svensson J, Appel L C, Blackwell B D, Dewar R L and Howard J 2010 Model data fusion: developing Bayesian inversion to constrain equilibrium and mode structure J. Plasma Fusion Res. 9 479-86

[19] Hole M J, von Nessi G, Svensson J, Appel L C and the MAST Team 2011 An equilibrium validation technique based on Bayesian inference Nucl. Fusion 51103005

[20] von Nessi G T, Hole M J and the MAST Team 2013 A unified method for inference of tokamak equilibria and validation of force-balance models based on Bayesian analysis J. Phys. A: Math. Theor. 46185501

[21] Svensson J and Werner A 2007 Large scale Bayesian data analysis for nuclear fusion experiments IEEE Int. Symp. on Intelligent Signal Processing (Alcala de Henares, Spain) pp 1-6

[22] von Nessi G T, Hole M J, Svensson J and Appel L 2012 Evidence cross-validation and Bayesian inference of MAST plasma equilibria Phys. Plasmas 19012506

[23] Dewar R L, Grimm R C, Johnson J L, Frieman E A, Greene J M and Rutherford P H 1974 Long wavelength kink instabilities in low pressure, uniform axial current, cylindrical plasmas with elliptic cross sections Phys. Fluids 17 930-8

[24] Cheng C Z, Chen L and Chance M S 1985 High-n ideal and resistive shear Alfvén waves in tokamaks Ann. Phys. $16121-47$

[25] Heidbrink W W 2002 Alpha particle physics in a tokamak burning plasma experiment Phys. Plasmas $92113-9$

[26] Bak J G, Lee S G and DeRac Son 2004 Performance of the magnetic sensor and the integrator for the KSTAR magnetic diagnostics Rev. Sci. Instrum. 75 4305-7

[27] Hole M J, Appel L C and Martin R 2009 A high resolution Mirnov array for the mega ampere spherical tokamak Rev. Sci. Instrum. 80123507

[28] Testa D and Albergante M 2012 Evidence for a new path to the self-sustainment of thermonuclear fusion in magnetically confined plasmas Europhys. Lett. 9735003

[29] de Kock L et al 1999 Design of the magnetic diagnostic for ITER Rev. Sci. Instrum. 70 452-7

[30] Vayakis G, Walker C, the ITER International Team, and Participant Teams 2003 Magnetic diagnostics for ITER/BPX plasmas Rev. Sci. Instrum. 74 2409-17

[31] Ambrosino G et al 2012 System-level optimisation of ITER magnetic diagnostics: preliminary results 24th IAEA Fusion Energy Conference (San Diego, CA, Oct 2012)

[32] John Wesson 2011 Tokamaks (The International Series of Monographs on Physics) 4th edn (Oxford: Oxford University Press)

[33] Sharapov S E et al and JET-EFDA Contributors 2004 Monitoring Alfvén cascades with interferometry on the JET tokamak Phys. Rev. Lett. 93165001

[34] Van Zeeland M A, Kramer G J, Nazikian R, Berk H L, Carlstromand T N and Solomon W M 2005 Alfvén eigenmode observations on DIII-D via two-colour $\mathrm{CO}_{2}$ interferometry Plasma Phys. Control. Fusion 47 L31-40

[35] Hacquin S, Sharapov S E, Alper B, Challis C D, Fonseca A, Mazzucato E, Meigs A, Meneses L, Nunes I, Pinches S D and JET EFDA Contributors 2007 Localized X-mode 
reflectometry measurements of Alfvén eigenmodes on the JET tokamak Plasma Phys. Control. Fusion 49 1371-90

[36] Kramer G J and Fu G-Y 2006 Reversed shear Alfvén eigenmodes associated with the ellipticity and triangularity Alfvén gaps Plasma Phys. Control. Fusion 48 1285-95

[37] Orsitto F P, Noterdaeme J M, Costley A E, Donné A J H and the ITPA TG on Diagnostics 2007 Requirements for fast particle measurements on ITER and candidate measurement techniques Nucl. Fusion 47 1311-7

[38] Bracewell R N 1986 The Fourier Transform and its Applications (New York: McGraw-Hill)

[39] van Milligen B Ph, Sanchez E, Estrada T, Hidalgo C, Brafias B, Carreras B and Garcia L 1995 Wavelet bicoherence: a new turbulence analysis tool Phys. Plasmas 2 3017-32

[40] Cohen L 1966 Generalized phase-space distribution functions Math. Phys. 7 781-6

[41] Choi H and Williams W J 1989 Improved time-frequency representation of multicomponent signals using exponential kernels IEEE Trans. Acoust. Speech Signal Process. 37 862-71

[42] Figueiredo A C A and Nave M F F 2004 Time-frequency analysis of nonstationary fusion plasma signals: a comparison between the Choi-Williams distribution and wavelets Rev. Sci. Instrum. 75 4268-70

[43] Merezhkin V G 1978 Sov. J. Plasma Phys. 4 152-64

[44] Klüber O et al 1991 MHD mode structure and propagation in ASDEX Nucl. Fusion 31 907-26

[45] Testa D, Fasoli A and Solano E 2003 Diagnosis and study of Alfvén eigenmodes stability in JET (invited) Rev. Sci. Instrum. 74 1694-700

[46] Schittenhelm M and Zohm H 1997 Analysis of coupled MHD modes with Mirnov probes in ASDEX Upgrade Nucl. Fusion 37 1255-70

[47] Nardone C 1992 Multichannel fluctuation data analysis by the singular value decomposition method. Application to MHD modes in JET Plasma Phys. Control. Fusion 34 1447-65

[48] Kim J S, Edgell D H, Greene J M, Strait E J and Chance M S 1999 MHD mode identification of tokamak plasmas from Mirnov signals Plasma Phys. Control. Fusion 41 1399-420

[49] Raju D, Jha R, Kaw P K, Mattoo S K, Saxena Y C and the ADITYA Team 2000 Mirnov coil data analysis for tokamak ADITYA Pramana J. Phys. 55 727-32

[50] Pretty D G and Blackwell B D 2010 A data mining algorithm for automated characterisation of fluctuations in multichannel timeseries Comput. Phys. Commun. 180 1768-76

[51] Hole M J, Hudson S R and Dewar R L 2007 Equilibria and stability in partially relaxed plasma-vacuum systems $\mathrm{Nucl}$. Fusion 47 746-53

[52] Bourguignon S, Carfantan H and Böhm T 2007 SparSpec: a new method for fitting multiple sinusoids with irregularly sampled data Astron. Astrophys. 462 379-87

[53] Press W H, Teukolsky S A, Vetterling W T and Flannery B P 1997 Numerical Recipes in Fortran 77: the Art of Scientific Computing 2nd edn (Cambridge: Cambridge University Press)

[54] Dudok de Wit T, Pecquet A L, Valet J C and Lima R 1994 The biorthogonal decomposition as a tool for investigating fluctuations in plasmas Phys. Plasmas 13288

[55] Castells F, Laguna P, Sörnmo L, Bollmann A and Roig J M 2007 Principal component analysis in ECG signal processing EURASIP J. Adv. Signal Process. 200774580

[56] Chatterjee A 2000 An introduction to the proper orthogonal decomposition Curr. Sci. 78 808-17

[57] Klein A, Carfantan H, Testa D, Fasoli A, Snpies J and JET EFDA Contributors 2008 A sparsity-based method for the analysis of magnetic fluctuations in unevenly-spaced Mirnov coils Plasma Phys. Control. Fusion 50125005

[58] Appel L C, Fulop T, Hole M J, Smith H M, Pinches S D, Vann R G L, and the MAST team 2008 Compressional Alfvén eigenmodes on MAST Plasma Phys. Control. Fusion 50115011

[59] Melrose D B and McPhedran R C 1991 Electromagnetic Processes in Dispersive Media (Sydney: Cambridge University Press)

[60] Hole M J, Robinson P A and Iver H Cairns 2004 Statistics of polarization and Stokes parameters of stochastic waves Phys. Rev. E 70036619

[61] Mendel J M 1991 Tutorial on higher-order statistics (spectra) in signal processing and system theory: theoretical results and some applications Proc. IEEE 79 278-305

[62] Fackrell J W A 1996 Bispectral analysis of speech signals PhD Thesis University of Edinburgh

[63] Maccarone T J and Schnittman J D 2005 The bicoherence as a diagnostic for models of high-frequency quasi-periodic oscillations Mon. Not. R. Astron. Soc. 357 12-6

[64] Raju D, Sauter O and Lister J B 2003 Study of nonlinear mode coupling during neoclassical tearing modes using bispectrum analysis Plasma Phys. Control. Fusion 45 369-78

[65] Itoh K, Nagashima Y, Itoh S-I, Diamond P H, Fujisawa A, Yagi M and Fukuyama A 2005 On the bicoherence analysis of plasma turbulence Phys. Plasmas 12102301

[66] White A E, Zweben S J, Burin M J, Carter T A, Hahm T S, Krommes J A and Maqueda R J 2006 Bispectral analysis of low- to high-confinement mode transitions in the National Spherical Tokamak Experiment Phys. Plasmas 13072301

[67] Austin D R, Hole M J, Robinson P A, Iver H Cairns and Dallaqua R S 2007 Laboratory evidence for stochastic plasma-wave growth Phys. Rev. Lett. 99205004

[68] Narita Y, Glassmeier K H, Décréau P M E, Hada T, Motschmann U and Nariyuki Y 2008 Evaluation of bispectrum in the wave number domain based on multi-point measurements Ann. Geophys. 26 3389-93

[69] Hole M J, Dallaqua R S, Simpson S W and Del Bosco E 2002 Plasma instability of a vacuum arc centrifuge Phys. Rev. E 65046409

[70] Robinson P A 1995 Stochastic wave growth Phys. Plasmas 21466

[71] Hole M J and Appel L C 2009 A modulation model for mode splitting of magnetic perturbations in the mega ampere spherical tokamak Plasma Phys. Control. Fusion 51045002

[72] Bak P, Tang C and Wiesenfeld K 1987 Self-organized criticality: an explanation for of $1 / f$ noise Phys. Rev. Lett. 59381

[73] Rickett B J 1990 Radio propagation through the interstellar medium Ann. Rev. Astron. Astrophys. 28561

[74] Robinson P A 1997 Nonlinear wave collapse and strong turbulence Rev. Mod. Phys. 69507

[75] Greenhough J, Chapman S C, Dendy R O and Ward D J 2003 Probability distribution functions for ELM bursts in a series of JET tokamak discharges Plasma Phys. Control. Fusion 45 747-58

[76] Webster A J and Dendy R O 2013 Statistical characterization and classification of edge-localized plasma instabilities Phys. Rev. Lett. 110155004

[77] Dendy R O, Chapman S C and Hnat B 2001 Sandpile model with tokamaklike enhanced confinement phenomenology Phys. Rev. Lett. 86 2814-7

[78] Rhee T, Kwon J M, Diamond P H and Xiao W W 2012 On the mechanism for edge localized mode mitigation by 
supersonic molecular beam injection Phys. Plasmas 19022505

[79] D'Ippolito D A, Myra J R and Zweben S J 2011 Convective transport by intermittent blob-filaments: comparison of theory and experiment Phys. Plasmas 18060501

[80] Garcia O E, Naulin V, Nielsen A H and Juul Rasmussen J 2005 Turbulence and intermittent transport at the boundary of magnetized plasmas Phys. Plasmas 12106209

[81] Pigarov A Yu, Krasheninnikov S I and Rognlien T D 2011 New approach in two-dimensional fluid modeling of edge plasma transport with high intermittency due to blobs and edge localized modes Phys. Plasmas 18092503

[82] Pankin A, McCune D, Andre R, Bateman G and Kritzÿ A 2004 The tokamak Monte Carlo fast ion module NUBEAM in the National Transport Code Collaboration library Comput. Phys. Commun. 159 157-84

[83] Hamilton B, McClements K G, Fletcher L and Thyagaraja A 2003 Field-guided proton acceleration at reconnecting X-points in flares Solar Phys. 214 339-52

[84] McClements K G and Hole M J 2012 Toroidal ripple transport of beam ions in the mega-ampére spherical tokamak Phys. Plasmas 19072514

[85] Tournianski M R, Akers R J, Carolan P G and Keeling D L 2005 Anisotropic fast neutral particle spectra in the MAST spherical tokamak Plasma Phys. Control. Fusion 47 671-84

[86] Luo Y, Heidbrink W W, Burrell K H, Kaplan D H and Gohil P 2007 Measurement of the $\mathrm{D}_{\alpha}$ spectrum produced by fast ions in DIII-D Rev. Sci. Instrum. 78033505

[87] Jones O M, Michael C A, McClements K G, Conway N J, Crowley B, Akers R J, Lake R J, Pinches S D and the MAST Team 2013 Fast-ion deuterium alpha spectroscopic observations of the effects of fishbones in the mega-ampere spherical tokamak Plasma Phys. Control. Fus. 55085009

[88] Zweben S J, Boivin R L, Diesso M, Hayes S, Hendel H W, Park H and Strachan J D 1990 Loss of alpha-like MeV Fusion Products from TFTR Nucl. Fusion 301551

[89] García-Muñoz M, Fahrbach H U, Zohm H and the ASDEX Upgrade Team 2009 Scintillator based detector for fast-ion losses induced by magnetohydrodynamic instabilities in the ASDEX Upgrade tokamak Rev. Sci. Instrum. 80053503

[90] Pace D C, Fisher R K, García-Munoz M, Darrow D S, Heidbrink W W, Muscatello C M, Nazikian R, Van Zeeland M A and Zhu Y B 2010 Modeling the response of a fast ion loss detector using orbit tracing techniques in a neutral beam prompt-loss study on the DIII-D tokamak Rev. Sci. Instrum. 81 10D305

[91] Perez von Thun C et al and JET EFDA contributors 2011 Numerical simulation of fast ion loss detector measurements for fishbones on JET Nucl. Fusion 51053003

[92] Berk H L and Breizman B N 1990 Saturation of a single mode driven by an energetic injected beam: III. Alfvén wave problem Phys. Fluids B 2 2246-52

[93] Heidbrink W W 2008 Basic physics of Alfvén instabilities driven by energetic particles in toroidally confined plasmas Phys. Plasmas 15055501

[94] Appel L C and Hole M J 2002 Modelling of Alfvénic activity in the MAST tokamak Proc. Joint Varenna-Lausanne Int. Workshop on the Theory of Fusion plasmas (Varenna, Italy, August)

[95] Pinches S D et al 1998 The HAGIS self-consistent nonlinear wave-particle interaction model Comput. Phys. Commun. $111113-49$
[96] Schneller M, Lauber Ph, Brudgam M, Pinches S D and Gunter S 2012 Double-resonant fast particle-wave interaction Nucl. Fusion 52103019

[97] Berk H L, Breizman B N, Fitpatrick J and Wong B N 1995 Line broadened quasi-linear burst model Nucl. Fusion 35 1661-8

[98] Hole M J, von Nessi G, Fitzgerald M and the MAST team 2013 Fast particle modifications to equilibria and resulting changes to Alfvén wave modes in tokamaks Plasma Phys. Control. Fusion 55014007

[99] Fülöp T, Pokol G, Helander P and Lisak M 2006 Destabilization of magnetosonic-whistler waves by a relativistic runaway beam Phys. Plasmas 13062506

[100] Fülöp T, Smith H M and Pokol G 2009 Magnetic field threshold for runaway generation in tokamak disruptions Phys. Plasmas 16022502

[101] Kómár A, Pokol G I and Fülöp T 2013 Electromagnetic waves destabilized by runaway electrons in near-critical electric fields Phys. Plasmas 20012117

[102] Chen K R, Huang R D, Wang J C and Chen Y Y 2005 Relativistic electromagnetic ion cyclotron instabilities Phys. Rev. E 71036410

[103] Zhang C, Zhang W, Lin Z and Li D 2013 Comparison of toroidicity-induced Alfven eigenmodes and energetic particle modes by gyrokinetic particle simulations Phys. Plasmas 20052501

[104] Vedenov A A 1962 Quasi-linear theory of a plasma At. Energ. 13 5-24

[105] Fülöp T, Pokol G and Lisak M 2008 Quasi-linear analysis of whistler waves driven by relativistic runaway beams in tokamaks Plasma Phys. Control. Fusion 50045003

[106] Besse N, Elskens Y, Escande D F and Bertrand P 2011 Validity of quasilinear theory: refutations and new numerical confirmation Plasma Phys. Control. Fusion 53025012

[107] Berk H L, Breizman B N and Pekker M 1996 Nonlinear dynamics of a driven mode near marginal stability Phys. Rev. Lett. 76 1256-9

[108] Lilley M K, Breizman B N and Sharapov S E 2009 Destabilizing effect of dynamical friction on fast-particle-driven waves in a near-threshold nonlinear regime Phys. Rev. Lett. 102195003

[109] Lilley M K, Breizman B N and Sharapov S E 2010 Effect of dynamical friction on nonlinear energetic particle modes Phys. Plasmas 17092305

[110] Lesur M and Diamond P H 2013 Nonlinear instabilities driven by coherent phase-space structures Phys. Rev. E 87031101

[111] Kosuga Y and Diamond P H 2012 Drift hole structure and dynamics with turbulence driven flows Phys. Plasmas 19072307

[112] Pinches S D, Berk H L, Gryaznevich M P, Sharapov S E and JET-EFDA Contributors 2004 Spectroscopic determination of the internal amplitude of frequency sweeping TAE Plasma Phys. Control. Fusion 46 S47

[113] Shinohara K et al and the ITPA Topical Group on Energetic Particles 2011 Effects of complex symmetry-breakings on alpha particle power loads on first wall structures and equilibrium in ITER Nucl. Fusion 51063028

[114] Farengo R, Ferrari H E, Firpo M-C, Garcia-Martinez P L and Lifschitz A F 2012 Alpha particle redistribution due to experimentally reconstructed internal kink modes Plasma Phys. Control. Fusion 54025007

[115] Gryaznevich M et al 1998 Achievement of record beta in the START spherical tokamak Phys. Rev. Lett. 80 3972-5

[116] Hole M J, von Nessi G, Fitzgerald M, McClements K G, Svensson $\mathrm{J}$ and the MAST Team 2011 Identifying the 
impact of rotation, anisotropy, and energetic particle physics in tokamaks Plasma Phys. Control. Fusion 53074021

[117] Fisch N J and Rax J-M 1992 Interaction of energetic alpha particles with intense lower hybrid waves Phys. Rev. Lett. $69612-5$

[118] Fisch N J 2007 Alpha channeling in mirror machines and tokamaks Fus. Sci. Technol. 51 1-6

[119] Ido T et al and the LHD experiment group 2012 Observation of a new energy channel from energetic particles to bulk ions through geodesic acoustic mode IAEA Fusion Energy Conf. (San Diego, CA, Oct 2012) PD/P8-16

[120] Sasaki M, Itoh K and Itoh S-I 2011 Energy channeling from energetic particles to bulk ions via beam-driven geodesic acoustic modes channeling Plasma Phys. Control. Fusion 53085017

[121] Donné A J H, Costley A E and Morris A W 2012 Diagnostics for plasma control on DEMO: challenges of implementation Nucl. Fusion 52074015 